\title{
IFI16 directly senses viral RNA and enhances RIG-I transcription and activation to restrict influenza virus infection
}

\author{
Zhimin Jiang $\oplus^{1,6}$, Fanhua Wei $\oplus^{2,6} \bowtie$, Yuying Zhang 3 , Tong Wang1, Weihua Gao1, \\ Shufang $\mathrm{Yu}^{2}$, Honglei Sun', Juan Pu', Yipeng Sun', Mingyang Wang'1, Qi Tong', Chengjiang Gao ${ }^{4}$, \\ Kin-Chow Chang $\oplus^{5}$ and Jinhua Liu ${ }^{1}$
}

\begin{abstract}
The retinoic acid-inducible gene I (RIG-I) receptor senses cytoplasmic viral RNA and activates type I interferons (IFN-I) and downstream antiviral immune responses. How RIG-I binds to viral RNA and how its activation is regulated remains unclear. Here, using IFI16 knockout cells and p204-deficient mice, we demonstrate that the DNA sensor IFI16 enhances IFN-I production to inhibit influenza A virus (IAV) replication. IFI16 positively upregulates RIG-I transcription through direct binding to and recruitment of RNA polymerase II to the RIG-I promoter. IFI16 also binds to influenza viral RNA via its HINa domain and to RIG-I protein with its PYRIN domain, thus promoting IAV-induced K63-linked polyubiquitination and RIG-I activation. Our work demonstrates that IFI16 is a positive regulator of RIG-I signalling during influenza virus infection, highlighting its role in the RIG-I-like-receptor-mediated innate immune response to IAV and other RNA viruses, and suggesting its possible exploitation to modulate the antiviral response.
\end{abstract}

\begin{abstract}
nfluenza virus is one of the most important causes of respiratory tract infection, resulting in at least 26 million influenza illnesses, 250,000 hospitalizations and 14,000 deaths during the 2019-2020 season in the United States (https://www.cdc.gov/flu/season/index. html). A(H1N1)/pdm2009, H3N2 and influenza B (B/Yamagata or $\mathrm{B} /$ Victoria lineage) viruses are mainly responsible for seasonal influenza epidemics each year. Moreover, avian influenza virus remains a notable additional threat to human health, in particular the H5, H7 and $\mathrm{H} 9$ subtypes. Although vaccination is the most effective way to control influenza A viruses (IAVs), prediction of evolving immunogenic epitopes as well as challenges in vaccine production and distribution often limit vaccine efficacy and availability. Furthermore, mutations in antiviral-resistant IAV strains continue to be identified, including the $\mathrm{H} 274 \mathrm{Y}$ mutation in neuraminidase (NA) ${ }^{1,2}$ and three major mutations (L26F, V27A and S31N) in the M2 protein ${ }^{3,4}$. Hence, the development of effective interventions against influenza virus infection remains an outstanding public health need. Targeting an essential host factor critical for influenza infection is a promising antiviral strategy ${ }^{5}$.

Several families of pattern recognition receptors have been described, including Toll-like receptors, retinoic acid inducible gene-I (RIG-I)-like receptors (RLRs) and nucleotideoligomerization-domain-like receptors, which are involved in the recognition of influenza viruses ${ }^{6-8}$. Among these, RIG-I is thought to be the most important sensor of influenza virus ${ }^{9}$; it binds the 5 'ppp-RNA of the virus, leading it to undergo conformational changes and exposing its caspase activation and recruitment domains $^{10}$, which is then ubiquitinated by the action of E3 ligases such as tripartite motif 25 (TRIM25) ${ }^{11}$ and RIPLET ${ }^{12}$. TRIM25
\end{abstract}

activates RIG-I through the generation of unanchored K63-linked polyubiquitin chains interacting with the caspase activation and recruitment domains ${ }^{13}$ or through the generation of anchored K63-linked polyubiquitin chains attached to K172 of RIG-I ${ }^{11}$. This process results in the interaction of RIG-I with the mitochondrial antiviral signalling (MAVS) adaptor, which leads to the subsequent activation of IRF3, IRF7 and NF- $\mathrm{KB}$ and thereby inducing the expression of type I interferon (IFN-I) and pro-inflammatory cytokines ${ }^{14}$. Therefore, RIG-I activation has to be tightly regulated to ensure effective virus inhibition while minimizing an excessive inflammatory response.

IFN- $\gamma$-inducible protein-16 (IFI16) is a member of the pyrin and HIN domain (PYHIN)-containing protein family, which encodes a class of homologous proteins that share a 200 -amino acid signature motif $(\mathrm{HIN})^{15}$. IFI16 was first reported as a sensor of transfected and viral DNA involved in innate signalling ${ }^{16,17}$, and it functions as an innate immune sensor in eukaryotic cells ${ }^{18-24}$. IFI16 senses double-stranded DNA from invading DNA viruses, including herpes simplex virus 1, Kaposi sarcoma-associated herpesvirus and vaccinia virus ${ }^{21,24,25}$, single-stranded DNA from HIV-infected $\mathrm{CD}^{4+}$ $\mathrm{T}$ cells and nuclear-damaged DNA from etoposide-treated keratinocytes ${ }^{22,26}$. DNA recognition by IFI16 induces activation of the stimulator of interferon genes-TANK-binding kinase 1-interferon regulatory factor 3 (STING-TBK1-IRF3) pathway, which leads to the induction of IFN-I or the ASC-caspase 1-dependent inflammasome to produce interleukin-1 $\beta$ (IL- $1 \beta)^{21,24,26}$. Additionally, a role for IFI16 in RNA virus infection has been identified, whereby IFI16 transcriptionally regulates the gene expression of IFN-I in Sendai virus infection ${ }^{27}$. The murine orthologue of IFI16 (p204) is

'Key Laboratory of Animal Epidemiology and Zoonosis, Ministry of Agriculture, College of Veterinary Medicine and State Key Laboratory of

Agrobiotechnology, China Agricultural University, Beijing, China. ${ }^{2}$ College of Agriculture, Ningxia University, Yinchuan, China. ${ }^{3}$ School of Biological Science and Technology, University of Jinan, Jinan, China. ${ }^{4}$ Key Laboratory of Infection and Immunity of Shandong Province and Department of Immunology, School of Biomedical Sciences, Shandong University, Jinan, China. ${ }^{5}$ School of Veterinary Medicine and Science, University of Nottingham, Sutton Bonington

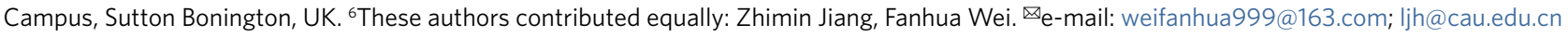


a

b
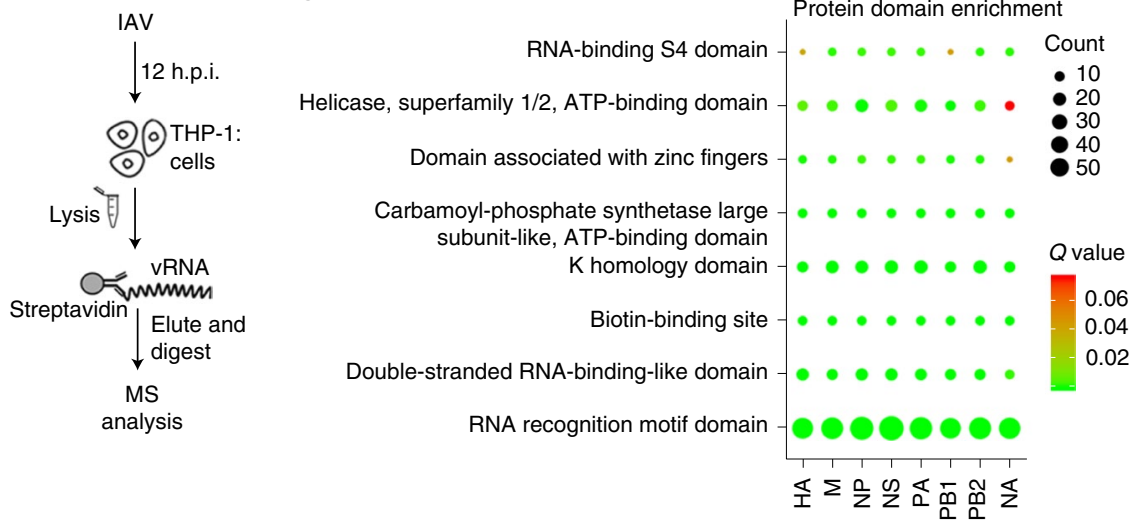

d
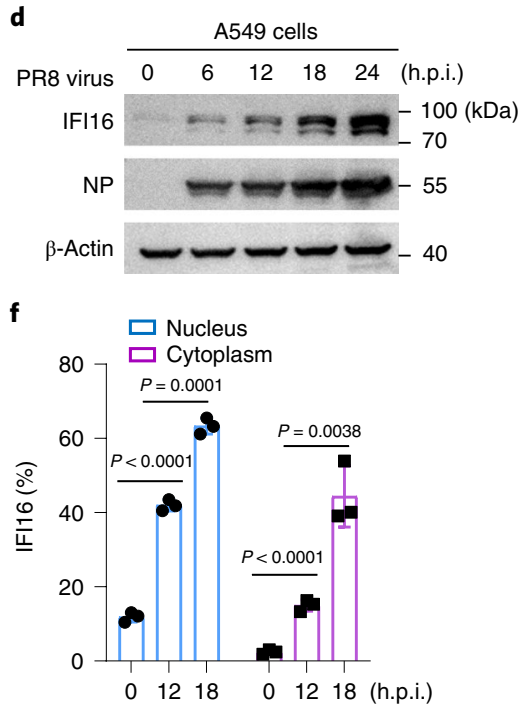

g

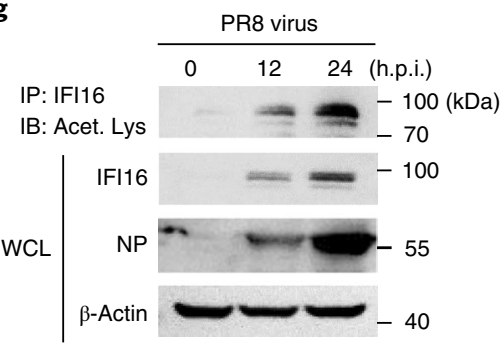

e $\quad$ h.p.i.

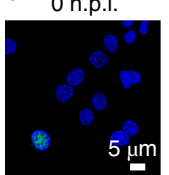

12 h.p.i.

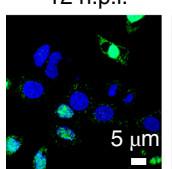

h

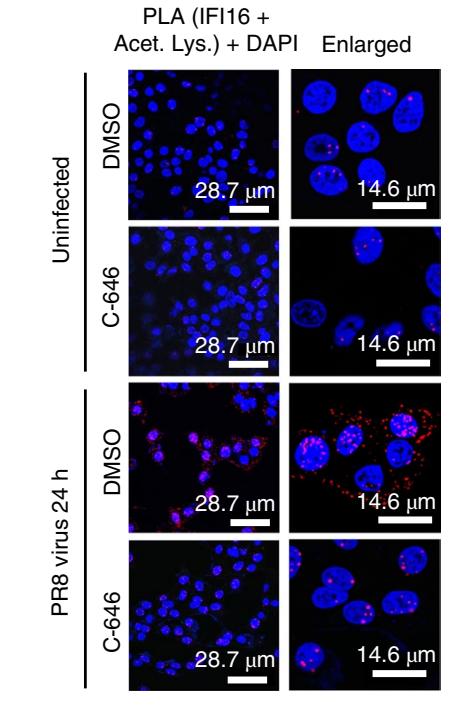

c Transcription level

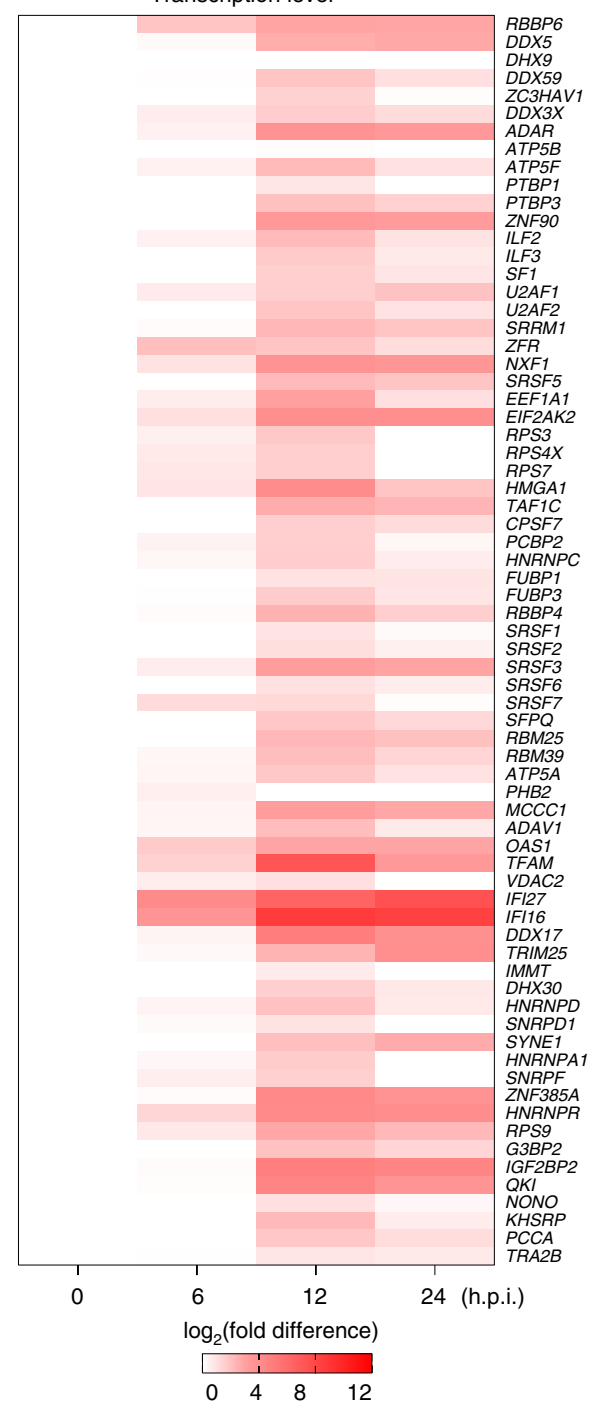

Fig. 1 | IFI16 is induced by IAV infection and is involved in the pathogenesis of virus infection. a, Schematic representation of the vRNA pull-down assay and MS approach to identify viral RBPs in THP-1 cells. b. Protein domain types significantly enriched among the human interacting proteins with indicated vRNA baits. The value range of $Q$ value is $[0,1]$. The closer $Q$ is to zero, the more significant the enrichment. Domains with $Q \leq 0.05$ are defined as domains that are significantly enriched in differentially expressed genes. c, mRNA expression levels, determined by RT-qPCR, of 70 candidate viral RBPs in THP-1 cells infected with PR8 virus at 2.0 m.o.i. RNA samplings were taken at 0, 6, 12 and 24 h.p.i. d, IFI16 protein expression in PR8-virus-infected (m.o.i. =1). A549 cells were evaluated by western blotting at $0,6,12,18$ and 24 h.p.i. ( $\beta$-actin detection was used as the loading control). e, Intracellular localization of IFI16 was assessed in PR8-virus-infected A549 cells at 0, 6, 12 and 18 h.p.i. by confocal microscopy. f, Quantification of the intracellular localization of IFI16 in cells as in e. The data represent the mean \pm s.d. ( $n=3$ independent experiments). $\mathbf{g}$, A549 cells were infected with PR8 virus at 0,12 and 24 h.p.i. Whole cell lysate (WCL) was then immunoprecipitated with anti-IFl16. Bound proteins were analysed by immunoblotting with anti-acetylated lysine (Acet. Lys). h, A549 cells were pre-incubated with C-646 for $2 \mathrm{~h}$, infected with PR8 virus for $1 \mathrm{~h}$, washed with PBS and incubated in complete medium with or without C-646. In situ PLA assays were used to assess IFI16 acetylation with anti-IFI16 and anti-acetylated lysine antibodies at 24 h.p.i. For $\mathbf{b}$, the results were assessed using parametric paired $t$-test (Student's one-tailed $t$-test). For $\mathbf{d}$-h, the data presented are representative of three independent experiments. Statistical significance in $\mathbf{f}$ was determined by unpaired two-tailed Student's $t$-test.

highly induced in mouse hepatitis coronavirus infection and inhibits IRF7-mediated IFN-I production ${ }^{28}$. Moreover, it was shown that IFI16 interacts with MAVS to promote the MAVS-mediated production of IFN-I that inhibits porcine reproductive and respiratory syndrome virus 2 replication ${ }^{29}$. More recently, IFI16 was found to directly bind chikungunya virus genome RNA and restrict viral replication and maturation ${ }^{30}$, which further suggests that IFI16 may play a crucial role in RNA virus infection. However, 
the precise role of IFI16 in influenza virus infection has not been elucidated.

Here, we show that IFI16, which was identified as an influenza viral RNA-binding protein (RBP), is highly induced both in vitro and in vivo during IAV infection. We further demonstrate that IFI16 upregulates $R I G-I$ transcription by binding its HINa domain to the RIG-I promoter, and interacts with both IAV viral RNA (vRNA) and RIG-I to promote influenza-virus-induced K63-linked polyubiquitination of RIG-I. Collectively, these results indicate that IFI16 is a key positive regulator of RIG-I signalling in antiviral innate immune responses to influenza virus infection.

\section{Results}

IFI16 is a viral RBP involved in influenza virus infection. Owing to its RNA genome, influenza virus utilizes RBPs of both viral and host origin for its replication. To comprehensively uncover vRNA-host protein interactions, we performed affinity purification coupled with mass spectrometry (AP-MS) analysis of influenza vRNA complexes. The eight vRNA segments of H7N9 virus were individually transcribed and labelled with biotin in vitro and then incubated with lysates from IAV-infected THP-1 cells. vRNA complexes bound to streptavidin magnetic beads were analysed by MS (Fig. 1a). Domain-enrichment analysis of co-purified proteins for each RNA bait revealed that more than $90 \%$ of the isolated proteins harboured nucleic-acid-binding domains (Fig. 1b). We identified 70 candidate viral RBPs that could bind to no fewer than 3 baits. A high number of co-purified proteins have been reported as RBPs, such as PTBP1 (ref. ${ }^{31}$ ), TRA2B ${ }^{32}$, DDX5 (ref. ${ }^{33}$ ), DDX3X ${ }^{34}$ and RBBP6 (ref. ${ }^{35}$ ), which indicates that our approach was effective in identifying vRNA-interacting proteins for influenza virus. The expression results demonstrated that IFI16 was most highly expressed for the duration of IAV infection (Fig. 1c), which suggests that IFI16 may play an important role in IAV infection.

To determine whether IFI16 is involved in IAV replication, we first evaluated IFI16 expression in PR8-virus-infected human cells. IFI16 mRNA levels were significantly upregulated in THP-1, A549 and HEK293 cells during PR8 infection (Extended Data Fig. 1a-c). We also found that PR8 infection significantly elevated p204 expression in mice lung tissues at 1 day post infection (d.p.i.) and at 3 and 5 d.p.i. (Extended Data Fig. 1d). PR8 virus actively induced the expression of IFI16 in THP-1 (Extended Data Fig. 1e) and A549 cells (Fig. 1d). Furthermore, ultraviolet-inactivated PR8 virus did not induce IFI16 expression in A549 cells compared with live virus infection (Extended Data Fig. 1f-g). Collectively, these findings suggest that IFI16 is involved in the modulation of influenza virus infection.

In uninfected cells, IFI16 was mainly localized in the nucleus, whereas PR8 infection induced an accumulation of IFI16 in the nucleus and cytoplasm (Fig. 1e,f). Consistent with the immunostaining results, PR8 virus infection caused a build-up of
IFI16 protein in the cytoplasm and nucleus (Extended Data Fig. 1g). Moreover, treatment of A549 cells with human IFN- $\gamma$ or polyinosinic:polycytidylic acid (poly(I:C)) induced IFI16 accumulation in the nucleus and cytoplasm (Extended Data Fig. 1i-k). It was reported that acetylation of IFI16 modulates its cellular distribution and cytoplasm translocation in DNA virus infection ${ }^{25,36}$. During IAV infection, we also detected the acetylation of IFI16 in PR8-virus-infected A549 cells at 12 and 24 hours post infection (h.p.i.) (Fig. $1 \mathrm{~g}$ and Extended Data Fig. 1l). Additionally, we performed in situ proximity ligation assays (PLAs), and low levels of nuclear acetylated IFI16 dots were detected in uninfected A549 cells (Fig. 1h). In contrast, acetylated IFI16 dots were clearly elevated in the nucleus and cytoplasm at 24 h.p.i. (Fig. $1 \mathrm{~h}$ ). We found that the IAV-induced accumulation of IFI16 in the cytoplasm was abolished in C-646-treated cells (Extended Data Fig. 1m). Collectively, IFI16 is highly upregulated during IAV infection and its associated acetylation can confer functional modifications such as stabilization of the IFI16 protein.

IFI16 inhibits IAV infection in vitro and in vivo. To determine the impact of IFI16 on influenza virus, IFI16-Flag vectors were transfected into A549 cells followed by PR8 infection at a multiplicity of infection (m.o.i.) of 1. Overexpression of IFI16 significantly reduced the viral titre of PR8 at 18 h.p.i. (Fig. 2a) and reduced the expression of viral NP and M1 proteins (Fig. 2b). Increasing the amounts of transfected IFI16-Flag vectors in HEK293T-Gluc cells resulted in impaired viral replication in a dose-dependent manner (Fig. 2c). In addition, overexpression of IFI16 inhibited the expression of mRNA and vRNA of NP and M1 genes in A549 cells (Extended Data Fig. 2a) and of protein levels of NP at 6,12 and 18h.p.i. in HEK293 cells (Extended Data Fig. 2b). Conversely, when IFI16 was knocked down by short interfering RNA (siRNA) in A549 cells, there were significant increases in viral protein and titres of progeny viruses (Extended Data Fig. 2c,d). To investigate the function of endogenous IFI16 during IAV infection, IFI16 $6^{+/+}$and $I F I 16^{-/-}$A549 cells were infected with PR8 virus. Infected $I F I 16^{-1-}$ A549 cells produced higher viral titres at 12 and 24h.p.i. (Fig. 2d) and protein levels of viral NP and M1 at 12 and 18 h.p.i. (Fig. 2e). In a gain-of-function experiment, exogenous expression of IFI16 in IFI16 ${ }^{-/}$A549 cells effectively reduced the expression of viral NP and M1 proteins at 12 and 18 h.p.i. (Fig. 2f). Accordingly, we found that the replication of green fluorescent protein (GFP)-tagged PR8 virus was markedly increased in $I F I 16^{-1-}$ A549 cells compared with $I F I 16^{+/+}$A549 cells (Fig. $2 \mathrm{~g}$ ). Collectively, these findings indicate that IFI16 inhibits influenza virus replication in human cells.

Additionally, PR8-infected wild-type (WT) mice suffered significantly less weight loss than $p 204$-deficient $\left(p 204^{-/-}\right)$mice, and they started to regain body weight by 8 d.p.i. (Fig. 3a). Moreover, survival rates of PR8 infected $p 204^{-/-}$mice were significantly poorer than infected WT mice (Fig. 3b), which suggests that

Fig. 2 | IFI16 expression inhibits IAV infection in vitro. a, A549 cells were transfected with IFl16-Flag plasmids or control for $24 \mathrm{~h}$ and then infected with PR8 virus at a m.o.i. of 1 for $18 \mathrm{~h}$. Viral titres were measured by $\mathrm{TCID}_{50}$ assays. Data are presented as the mean \pm s.e.m. and are representative of three independent experiments. b, Left: A549 cells were transfected with IFl16-Flag plasmids or control for $24 \mathrm{~h}$ and then infected with PR8 virus at a m.o.i. of 1 , and NP and M1 proteins were detected. Right: data were quantified and shown as the ratio of NP to $\beta$-actin and M1 to $\beta$-actin. The data represent the mean \pm s.e.m. $(n=3)$. c, HEK293T-Gluc cells were transfected with different amounts of IFl16-Flag or control, followed by infection with PR8 virus $(m .0 . i .=1)$ for $24 \mathrm{~h}$. Viral infectivity was determined. Data are presented as the mean \pm s.e.m. and are representative of three independent experiments. d, Viral titres in PR8-virus-infected (m.o.i. =1) IFI16 ${ }^{+/+}\left(\mathrm{IFI16} \mathrm{WT)}\right.$ and IFI16 ${ }^{-/-}(\mathrm{IFI16} \mathrm{KO})$ A549 cells were measured by TCID 50 assay. Data are presented as the mean \pm s.e.m. and are representative of three independent experiments. e, Left: the NP and M1 protein expression levels in PR8-virus-infected (1 m.o.i.) $I F I 16^{+/+}$and IFI16-/- A549 cells were analysed. Right: data were quantified and shown as the ratio of NP to $\beta$-actin and M1 to $\beta$-actin. The data represent the mean \pm s.e.m. $(n=3)$. f, Left: IFI16 $6^{+/+}$and IFI16 ${ }^{-/-}$A549 cells were transfected with IF/16-Flag plasmids or empty control for $24 \mathrm{~h}$ and then infected with PR8 virus at a m.o.i. of 1. NP and M1 proteins in virus-infected A549 cells were detected. Right: data were quantified and shown as the ratio of NP to $\beta$-actin and $\mathrm{M} 1$ to $\beta$-actin. The data represent the mean \pm s.e.m. $(n=3)$. g, Fluorescence microscopy images of viral replication (green) in IFl16 $6^{+/+}$and IF/16 $6^{-/-}$A549 cells after infection with GFP-PR8 virus for $12 \mathrm{~h}$. For $\mathbf{b}$ and $\mathbf{d}-\mathbf{g}$, data are representative of three independent experiments. Statistical significance in $\mathbf{a}-\mathbf{f}$ was determined by unpaired two-tailed Student's $t$-test. 
control of IAV infection in vivo also requires p204. In lung tissues of $p 204^{-1-}$ mice, PR8 virus replication at 4 and 6 d.p.i. was higher (Fig. $3 c$ ), with accompanying greater viral NP mRNA and vRNA levels (Fig. 3d), in lung tissues of $p 204^{-/-}$mice than in corresponding
WT mice. Moreover, viral NP staining was more intense in lung sections of $p 204^{-1-}$ mice at 5 d.p.i. than in corresponding WT mice (Fig. 3e). Notably, gross pathology and histopathology analyses revealed that lung tissues of PR8-virus-infected $p 204^{-/-}$mice a

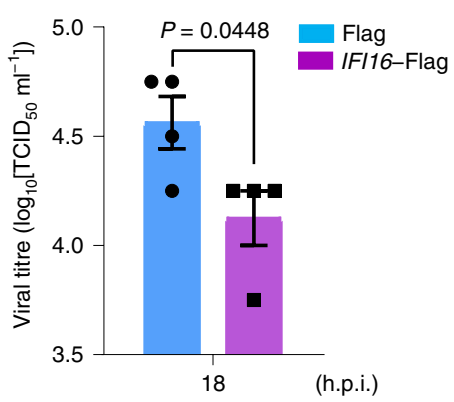

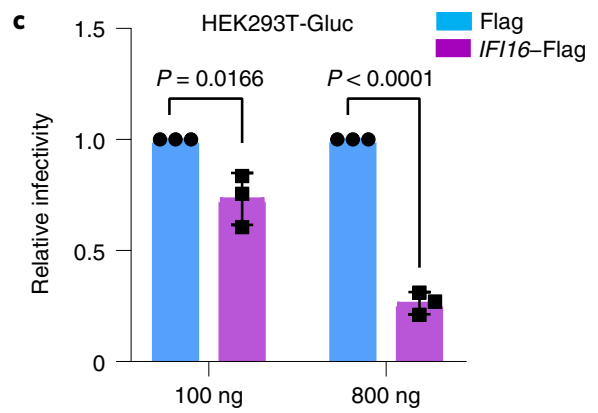

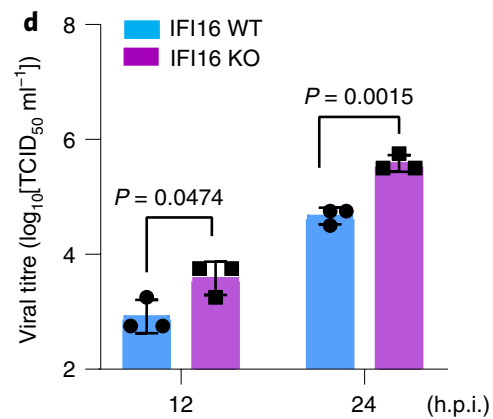

b

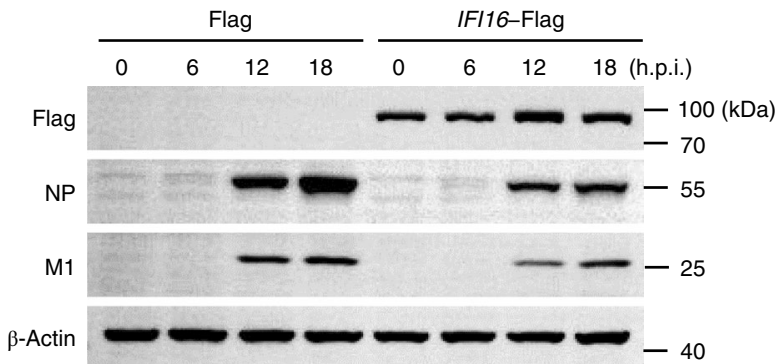

e

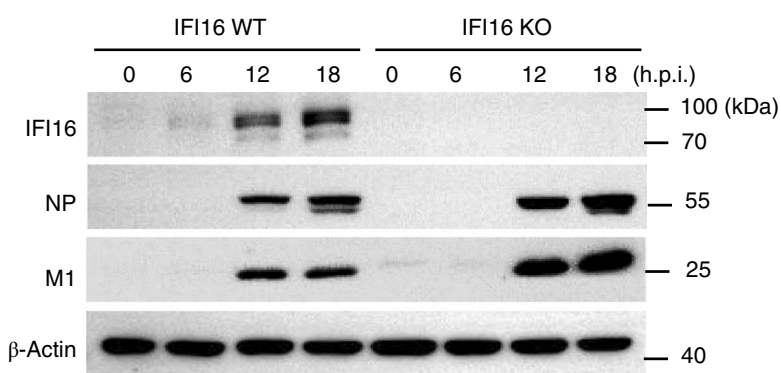

$\mathbf{f}$
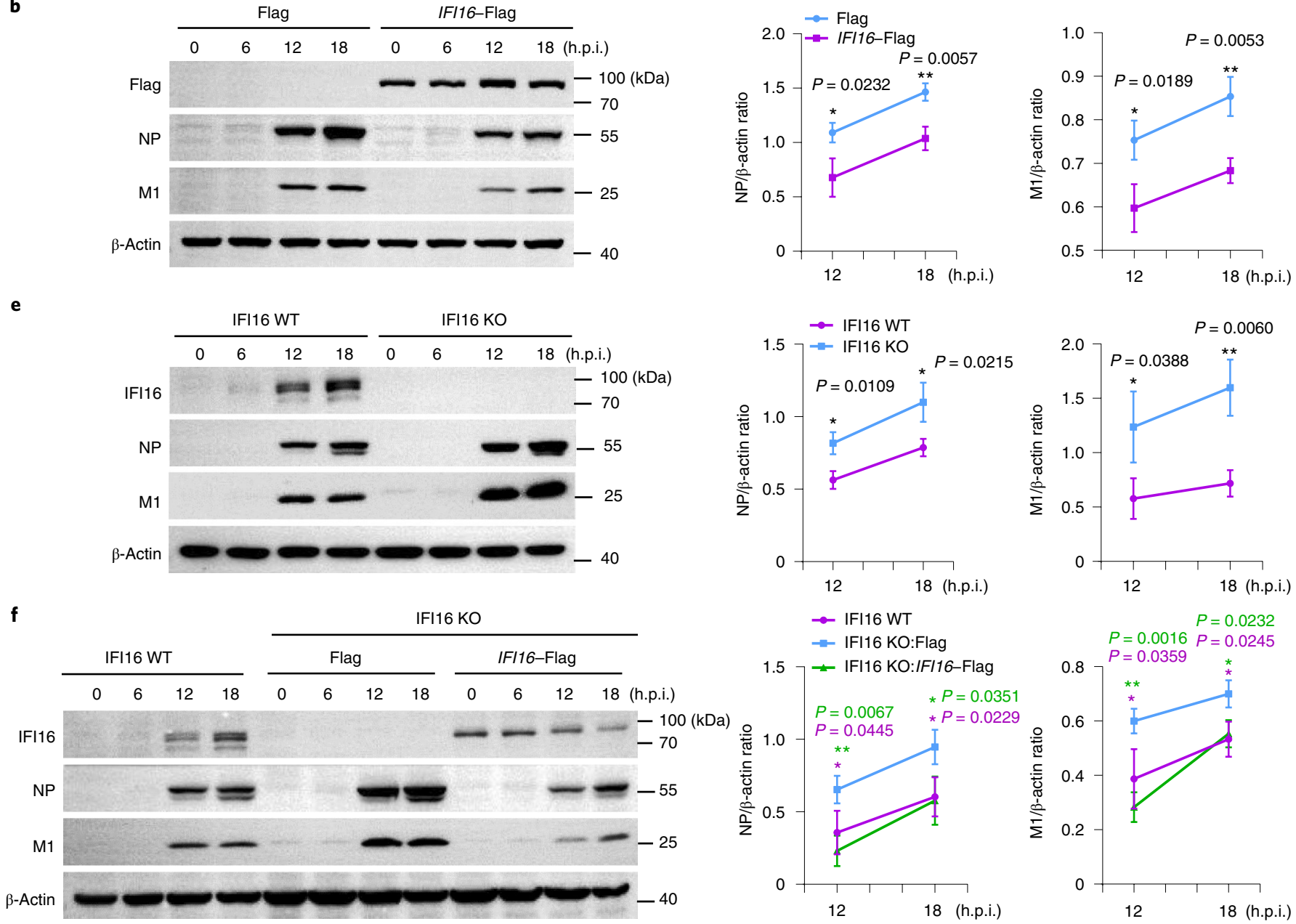

g

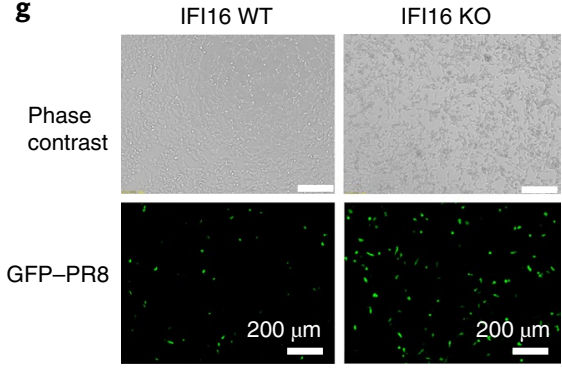

IFI16 KO 
a

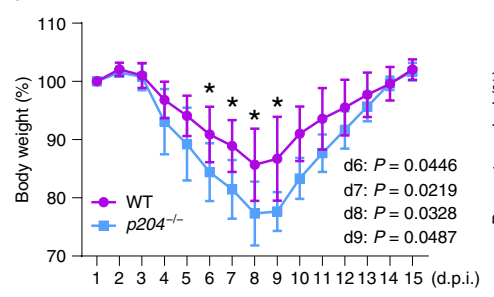

b

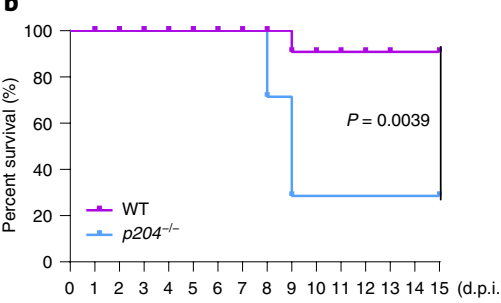

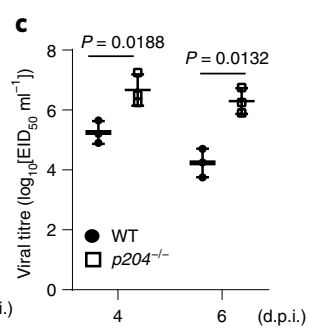
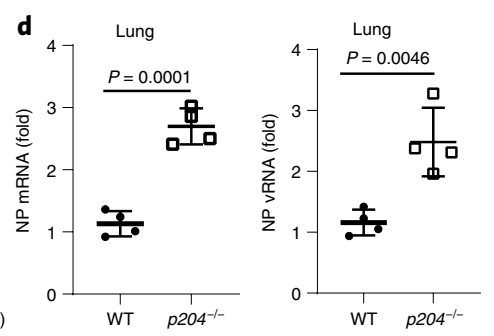

e
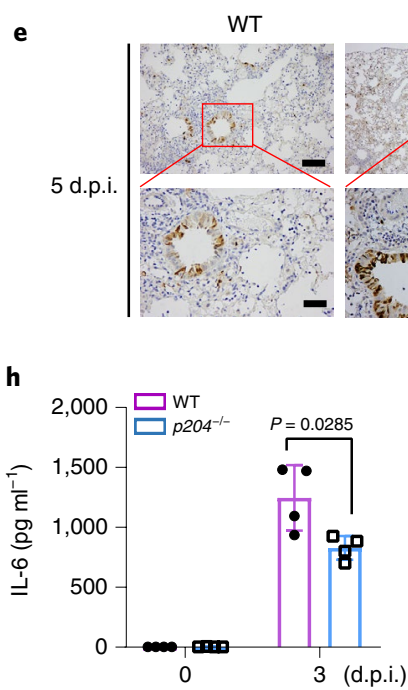

I

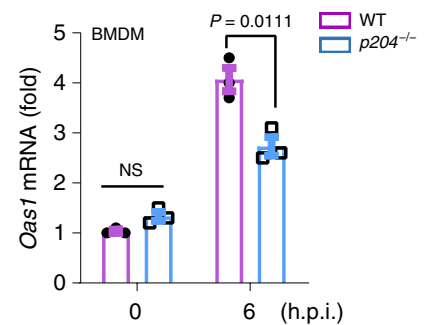

p204-/-

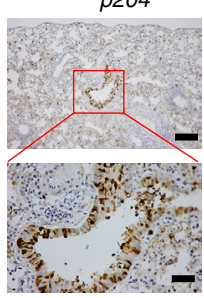

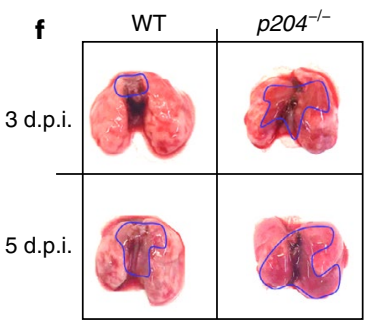

j
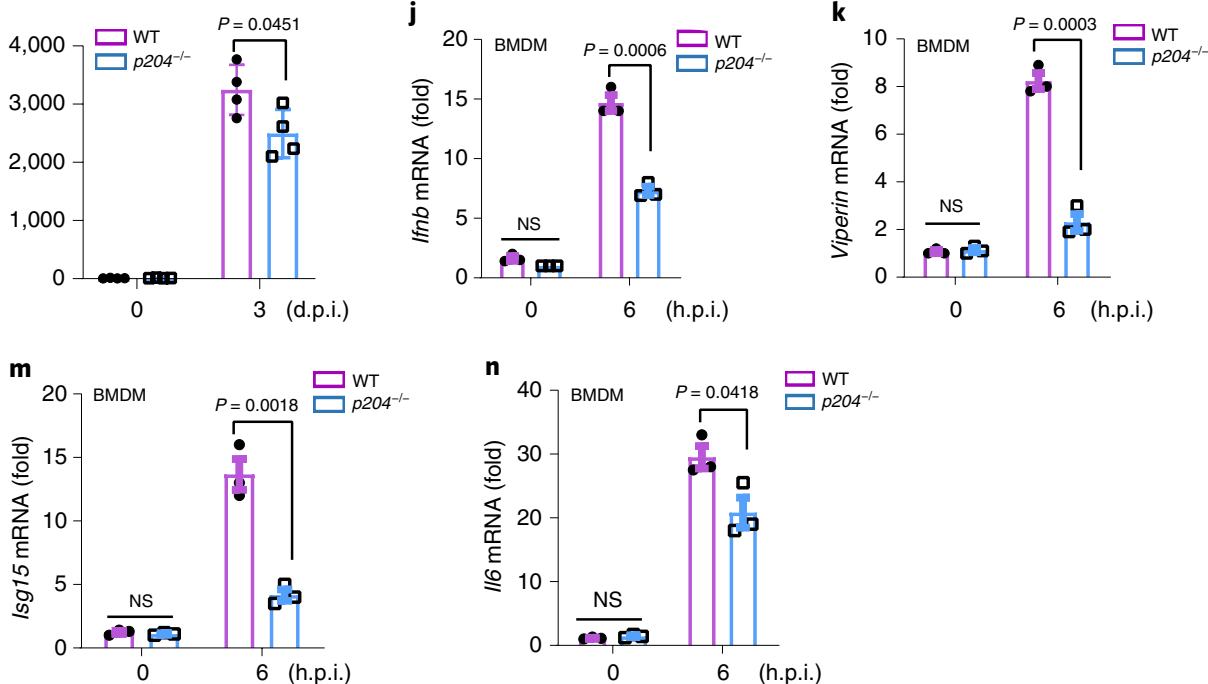

g

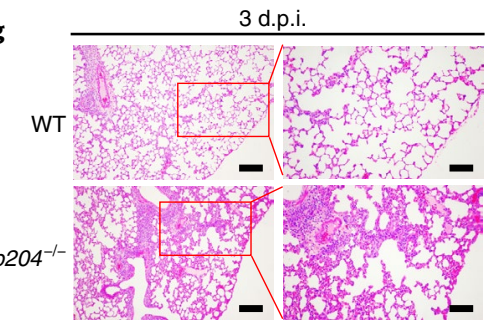

i
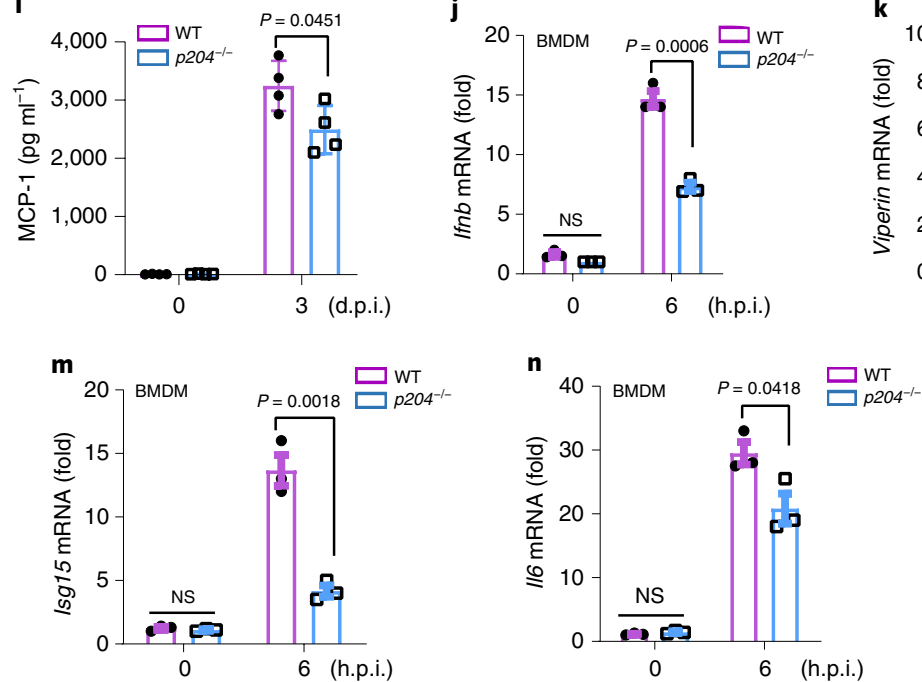

Fig. 3 | p204-deficient mice are susceptible to IAV infection. a, WT and $p 204^{-/-}$mice $(n=6)$ were infected with 50 TCID 50 of PR8 virus. Changes in body weight were monitored daily. Data are presented as the mean \pm s.d. b. Survival of WT and p204-/- mice infected with 50 TCID 50 of PR8 virus. Data presented were pooled from three independent experiments. Kaplan-Meier survival curves were compared using log-rank (Mantel-Cox) analysis. c, Viral titres in lung tissues from $50 \mathrm{TCID}_{50}$ of PR8-virus-infected WT and p204-/- mice at 4 and 6 d.p.i. were determined by EID 50 assay. Data are from three independent experiments with $n=6$ mice per group run in triplicate. Error bars indicate s.e.m. d, Viral NP mRNA (left) and vRNA (right) in lung tissues of PR8-virus-infected WT and $p 204^{-/-}$mice at 3 d.p.i. were determined by RT-qPCR. Data are presented as the mean \pm s.e.m. and are representative of three independent experiments. e, Viral NP protein expression in lung tissue sections from virus-infected WT and p204 ${ }^{-/-}$mice ( $n=3$ ) mice was examined by immunohistochemistry. Representative sections of one mouse out of three are shown. Scale bars, $800 \mu \mathrm{m}$ (top) and $80 \mu \mathrm{m}$ (bottom). $\mathbf{f}$, Images of gross lesions (outlined in blue) of lung tissues from virus-infected WT and p204-/- mice at 3 and 5 d.p.i. Representative sections of one mouse out of three are shown. g, H\&E staining of lung tissues from virus-infected WT and p204-/- mice at 3 d.p.i. Scale bars, $600 \mu \mathrm{m}$ (left) and $120 \mu \mathrm{m}$ (right). Representative H\&E staining images are from six mice per group of three independent experiments. h,i, IL-6 and MCP-1 levels in BALF from virus-infected WT and p204 ${ }^{-/-}$mice $(n=4)$ were quantified. Data are presented as the mean \pm s.d. $\mathbf{j}-\mathbf{n}$, mRNA expression of Ifnb (j), viperin ( $\left.\mathbf{k}\right)$, Oas1 (I), Isg15 ( $\left.\mathbf{m}\right)$ and $/ 16$ ( $\left.\mathbf{n}\right)$ in BMDMs of virus-infected WT and $p 204^{-/-}$mice was determined by RT-PCR. Data presented as mean \pm s.e.m. and are representative of three independent experiments. Statistical significance in $\mathbf{a}, \mathbf{c}, \mathbf{d}$ and $\mathbf{h}-\mathbf{n}$ was determined by unpaired two-tailed Student's $t$-test.

displayed more extensive damage at 3 and 5 d.p.i. (Fig. $3 \mathrm{f}$ ), and more severe inflammatory damage at 3 d.p.i. (Fig. $3 g$ ), than in corresponding WT mice. Accordingly, IL- 6 and MCP-1 protein levels in bronchoalveolar fluid (BALF) were also significantly higher in PR8-infected WT mice than those found in corresponding $p 204^{-1-}$ mice (Fig. 3h,i). Furthermore, p204 deficiency dramatically inhibited the transcription of Ifnb, Viperin, Oas1, Isg15 and Il6 in PR8-virus-infected bone marrow-derived macrophages (BMDMs) (Fig. $3 j-n$ ). Therefore, IFI16 is required for host defence against influenza virus infection in vitro and in vivo.
IFI16 enhances the production of IFN-I in IAV infection. DNA recognition by IFI16 induces the activation of the STING-TBK1IRF3 pathway, which leads to the induction of IFN-I ${ }^{21,24,26}$. To investigate the mechanism of innate immune activation by IFI16, we performed transcriptomics analysis in $I F I 16^{+/+}$and $I F I 16^{-1-}$ A549 cells infected with PR8 virus for $12 \mathrm{~h}$. In the Gene Ontology (GO) term enrichment analysis, ten enriched terms identified were related to antiviral responses (Fig. 4a). The number of genes associated with individual terms and enrichment probability were lower in FFI16 $^{-1-}$ A549 cells (Fig. 4a). Sixteen IFN-stimulated genes (ISGs) 
were exclusively upregulated in $I F I 16^{+/+}$cells during IAV infection, while only 4 ISGs were upregulated in IFI16 $6^{-1-}$ A549 cells (Fig. 4b). Collectively, these results show that the induction of ISGs is reduced in the absence of IFI16. We next evaluated the function of IFI16 in the induction of IFN-I. Quantitative PCR with reverse transcription (RT-qPCR) analysis showed that overexpression of IFI16 enhanced PR8-virus-induced expression of IFNB, ISG15 and IL6 at 6 and 12 h.p.i. (Extended Data Fig. 3a-c). Conversely, IFI16 deficiency greatly reduced the PR8-virus-induced expression of IFNB, RIG-I, ISG56 and viperin (Fig. 4c-f) as well as IFNA4, IRF7 and CXCL5 (Extended Data Fig. 3d-f) in A549 cells. Furthermore, overexpression of IFI16 in A549 cells increased PR8-virus-induced IFN- $\beta$ protein production at 12 and 18 h.p.i. in a dose-dependent manner (Fig. 4g), while IFI16 deficiency markedly decreased IFN- $\beta$ production (Fig. 4h). These results suggest that IFI16 mediates antiviral effects through influenza-virus-induced IFN-I signalling.

To examine the contribution of IFI16 to RIG-I signalling, we transfected IFI16-Flag or control plasmids into A549 cells followed by infection with PR8 virus at 1 m.o.i. Overexpression of IFI16 in infected cells enhanced protein detection of RIG-I, phosphorylated TBK1 (p-TBK1), p-IRF3 and p-P65 relative to infected controls (Fig. 4i). In contrast, IFI16 deficiency in infected cells led to reduced protein detection of RIG-I, p-TBK1 and p-IRF3 compared with the IFI16 $6^{+/+}$group (Fig. 4j). Consistent with these results, overexpression of IFI16 in the presence of 5'ppp-RNA also increased protein detection of RIG-I, p-TBK1 and p-IRF3 (Extended Data Fig. 3g). Furthermore, exogenous expression of IFI16 in infected IFI16 ${ }^{-1-}$ A549 cells restored protein expression of RIG-I, p-TBK1 and p-IRF3 (Extended Data Fig. 3h). The immunofluorescence results showed that IFI16 deficiency reduced the level of p-IRF3 (Ser396) in the nucleus in PR8-virus-infected A549 cells (Fig. 4k,1). Taken together, these findings indicate that IFI16 is a potent stimulator of the IFN-I response in influenza virus infection.

IFI16 transcriptionally upregulates the expression of RIG-I. Overexpression of IFI16 progressively upregulated RIG-I protein expression in A549 cells in a time-dependent manner (Fig. 5a) and in a dose-dependent manner (Extended Data Fig. 4a). HEK293 cells co-transfected with IFI16-Myc and RIG-I-Flag followed by cycloheximide treatment did not exhibit evidence of RIG-I protein degradation (Extended Data Fig. 4b). Overexpression of IFI16 also increased the protein and mRNA expression of RIG-I in IFNAR1 $1^{-1-}$ A549 cells in a dose-dependent manner (Extended Data Fig. 4c,d). Furthermore, A549 cells transfected with IFI16Flag vectors showed rising levels of RIG-I mRNA in a vector dose-dependent manner (Extended Data Fig. 4e) and in a progressive time-dependent manner (Fig. 5b). Consistent with the findings in A549 cells, mRNA expression of RIG-I in PR8-virus-infected lung tissues of $p 204$-deficient mice was significantly reduced at 1 and 3 d.p.i. compared with corresponding WT mice (Extended Data Fig. 4f). Taken together, our results indicate that IFI16 upregulates $R I G-I$ at the transcriptional level.

To further assess the role of IFI16 in the regulation RIG-I, a luciferase reporter vector driven by the RIG-I promoter was used in transfections of HEK293 cells. The RIG-I promoter was responsive to IFI16 overexpression in a vector dose-dependent manner (Fig. 5c), which indicates that IFI16 is capable of transactivating the RIG-I promoter.

To determine the minimum promoter length of $R I G-I$ that is responsive to IFI16 induction, an extensive series of $R I G-I$ promoter deletion constructs, each spliced to the luciferase reporter gene, was generated for co-transfections with increasing amounts of IFI16-Flag expression vectors (Fig. 5d-i). The minimum RIG-I promoter responsive to IFI16-Flag overexpression was between -371 and $-360 \mathrm{bp}$ in length (Fig. 5i). Furthermore, Flag antibody pull-down experiments, based on a series of biotinylated double-stranded DNA probes that spanned the minimum promoter section (Extended Data Fig. 4g), showed that only probe p2-mut4, which harboured mutations between the -371 and -360 region of the promoter, failed to bind IFI16-Flag (Extended Data Fig. 4h). Chromatin immunoprecipitation (ChIP) and qPCR assays, using two sets of primer pairs that target the -500 to $-250 \mathrm{bp}$ region of the $R I G-I$ promoter, further demonstrated direct binding of IFI16 to the RIG-I promoter (Fig. $5 \mathrm{j}$ ). Collectively, these results show that IFI16 is capable of binding to the RIG-I promoter to promote $R I G-I$ transcription.

Next, to determine whether IFI16 affects the recruitment of RNA polymerase II (Pol II) to the RIG-I promoter, ChIP was performed with an anti-Pol II antibody on IFI16 ${ }^{+/+}$and $I F I 16^{-/-}$A549 cells infected with PR8 virus at 1 m.o.i. for 0 and $12 \mathrm{~h}$. qPCR targeting the detection of the basal promoter site of RIG-I showed more RNA Pol II binding to the basal promoter of infected $I F I 16^{+/+}$than infected IFI16 ${ }^{-1-}$ A549 cells (Fig. 5k), but no significant difference for the GAPDH promoter (Extended Data Fig. 4i), which suggests that during infection, IFI16 facilitates RNA Pol II recruitment to the basal promoter of RIG-I.

To investigate the domain(s) in IFI16 responsible for activating RIG-I transcription, we constructed IFI16 expression mutants bearing different domain deletions (Extended Data Fig. 4j). Mutants without the HINa domain completely lost the ability to activate the RIG-I-luc reporter gene (Fig. 5l). In a separate experiment, we also found that in the absence of the HINa domain, there was no binding of IFI16 to a biotinylated RIG-I promoter (Extended Data Fig. 4k). Deletion of the HINa domain in IFI16 abrogated its antiviral activity in response to PR8 virus infection (Extended Data Fig. 4l). Taken together, these data show that IFI16 binds the RIG-I promoter with its HINa domain facilitating the recruitment of RNA Pol II to the site, thereby enhancing the transcriptional activation of RIG-I.

Fig. 4 | IFI16 enhances RIG-I-mediated production of IFN-I during IAV infection. a, GO term analysis of upregulated host proteins in IAV-infected IF/16 ${ }^{+/+}$ and IFI16-/- A549 cells. The top ten GO terms are ordered by enrichment $P$ values. The $P$ values indicated in the legend and indices in cells correspond to the number of protein groups associated with individual $\mathrm{GO}$ terms in respective comparisons. b, Differential expression of host genes between IAV-infected and mock conditions in $I F I 16^{+/+}\left(x\right.$ axis) and $I F I 16^{-/-}(y$ axis). Proteins exhibiting significant differential responses are highlighted in green (ISGs) or orange (non-ISGs). c-f, Gene expression levels of IFNB (c), RIG-I (d), ISG56 (e) and Viperin (f) in PR8-virus-infected IFI16 $6^{+/+}$and IFI16 $6^{-/-}$A549 cells at 0,6 and 12 h.p.i. were determined by qPCR. $\mathbf{g}$, A549 cells were transfected with IFl16-Flag expression vectors or empty vector (EV) for $24 \mathrm{~h}$ and then infected with PR8 virus. IFN-I levels in supernatants were quantified using IFN bioassays at 2, 12, 18, 24 and 36 h.p.i. at $1 \mathrm{~m}$.o.i. h, IFI16 ${ }^{+/+}$and IFI16 ${ }^{-1-}$ A549 cells were infected with PR8 virus at 1 m.o.i. IFN-I levels in supernatants were then measured by IFN bioassays at $2,12,18,24$ and 36 h.p.i. i, A549 cells were transfected with IF/16-Flag expression vectors or empty control for $24 \mathrm{~h}$ and then infected with $1 \mathrm{~m}$.0.i. of PR8 virus for 0, 2, 4, 8 and $12 \mathrm{~h}$. RIG-I-triggered downstream signalling molecules were evaluated using the indicated antibodies. $\mathbf{j}$, RIG-I-triggered downstream signalling molecules in PR8-virus-infected IFI16 ${ }^{+/+}$and IFI16 $6^{-/-}$A549 cells at 0, 4, 8 and 12 h.p.i. were analysed using the indicated antibodies. k, IFI16 $6^{+/+}$and $I F / 16^{-/-}$A549 cells were infected with PR8 virus at 5 m.o.i. Nuclear localization of p-IRF3 was then determined by p-IRF3 (Ser396) intracellular immunostaining for confocal microscopy. I, Quantification of nuclear localization of p-IRF3 (Ser396). For i-k, data are representative of three independent experiments. For $\mathbf{a}$, the results were assessed using parametric paired $t$-test (Student's one-tailed $t$-test). For $\mathbf{c}-\mathbf{h}$ and $\mathbf{I}$, data are presented as the mean \pm s.d. from three independent experiments, and the significance of the results was assessed using parametric paired $t$-test (Student's two-tailed $t$-test). 
IFI16 binds vRNAs and interacts with RIG-I. Since IFI16 is also a RBP, we determined whether IFI16 binds vRNAs as with the RIG-I promoter. Pull-down assays using cell lysates of HEK293 cells, separately overexpressing Flag-tagged IFI16, RIG-I, MDA-5 and TBK1 proteins, incubated with biotinylated NP vRNA showed binding of IFI16, RIG-I and MDA-5 to the vRNAs (Extended Data Fig. 5a). Microscale thermophoresis technology (MST) results showed that the HINa domain also exhibited high affinity for full-length NS vRNA (Extended Data Fig. 5b). To further demonstrate binding between IFI16 and other IAV RNAs, purified glutathione $S$-transferase
(GST)-IFI16 proteins were incubated with fluorescein-labelled HA, NP, PA and PB2 vRNAs. MST assays also showed IFI16 binding with the different vRNAs (Extended Data Fig. 5c). Furthermore, RNA fluorescence in situ hybridization (FISH) analysis revealed increasing colocalization of IFI16 with NP vRNA in PR8-virus-infected A549 cells at 6 and 12 h.p.i. (Fig. 6a). To identify bound vRNAs during infection, RNA eluted from co-precipitation of IFI16-Flag from IAV-infected $R I G-I^{-1-}$ cells was subjected to deep sequencing analysis, which detected IFI16 binding to all eight IAV gene segments (Fig. 6b). RIG-I has been found with IAV genomic fragments ${ }^{37}$. IFI16

\begin{tabular}{r|cc|} 
& No. WT & No. KO \\
\cline { 2 - 3 } IFN-I signalling pathway & 18 & 11 \\
Defence response to virus - & 35 & 18 \\
Response to virus - & 23 & 14 \\
Immune response - & 58 & 13 \\
Immune system process - & 41 & 13 \\
Innate immune response - & 49 & 22 \\
Response to IFN- $\beta-$ & 4 & 3 \\
Negative regulation of viral genome replication - & 22 & 13 \\
Positive regulation of immune response - & 4 & 3 \\
Cellular response to IFN- $\alpha-$ & 3 & 2 \\
\hline
\end{tabular}

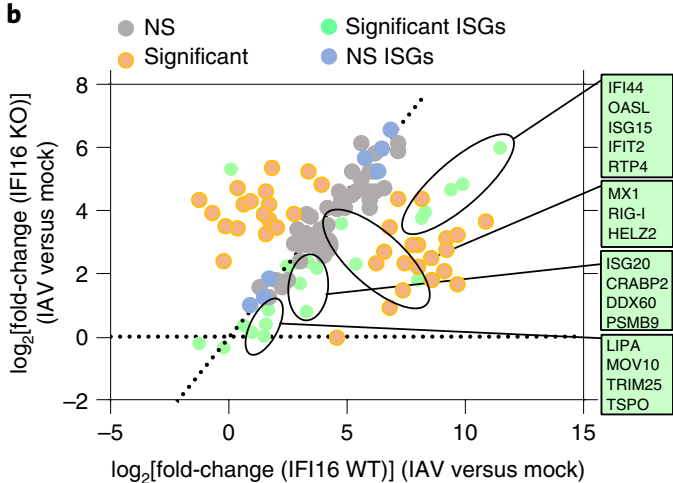

$\log _{2}$ [fold-change (IFI16 WT)] (IAV versus mock) c

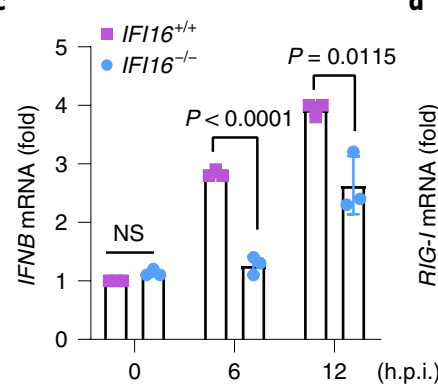

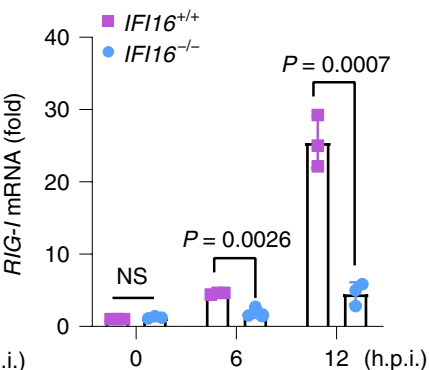

e

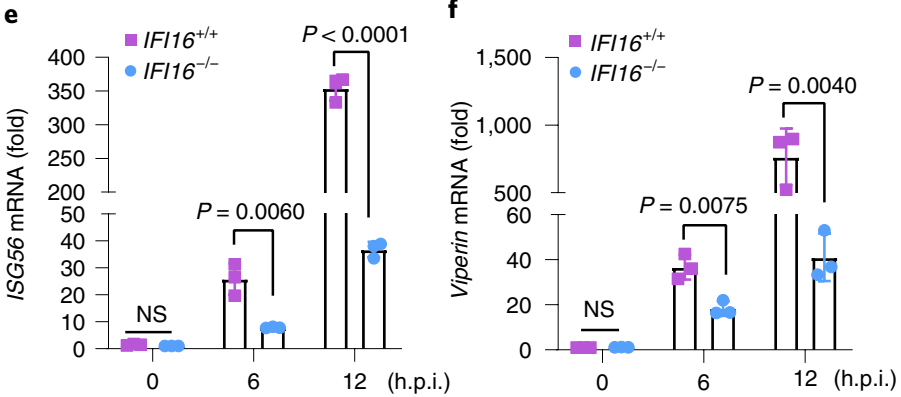

g

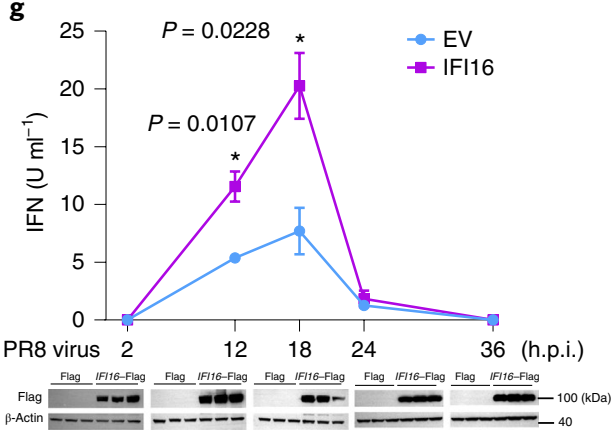

h

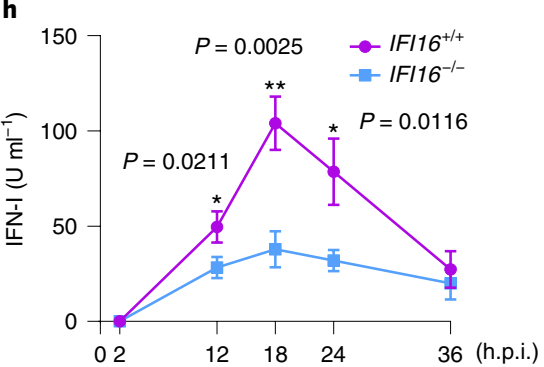

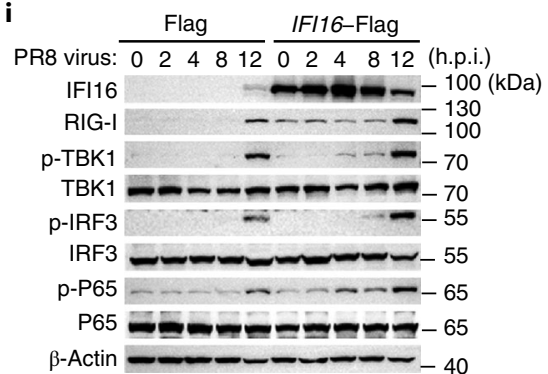

j

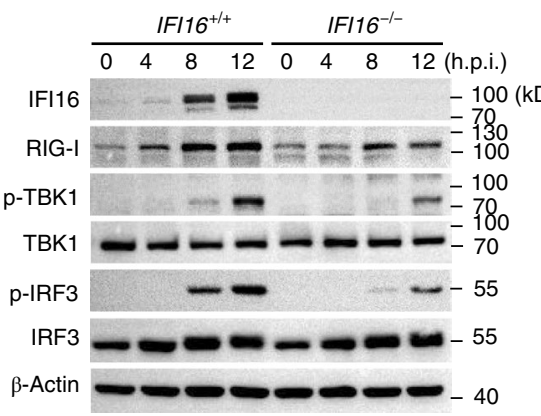

$\mathbf{k}$

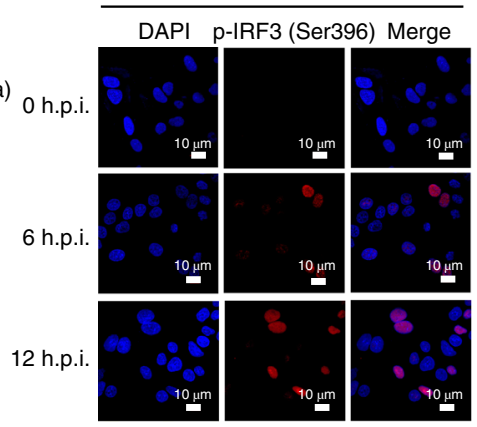

DAPI p-IRF3 (Ser396) Merge

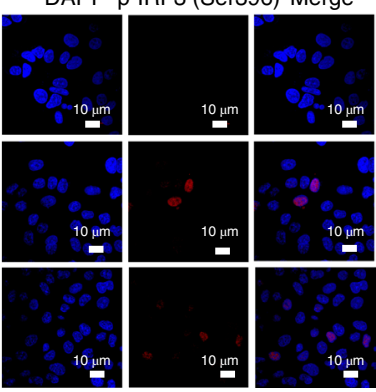

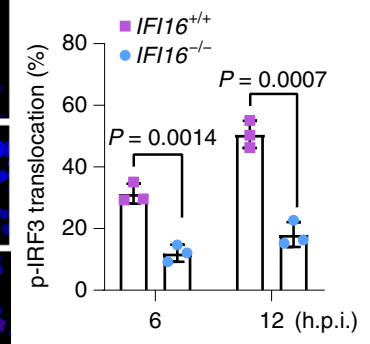


a

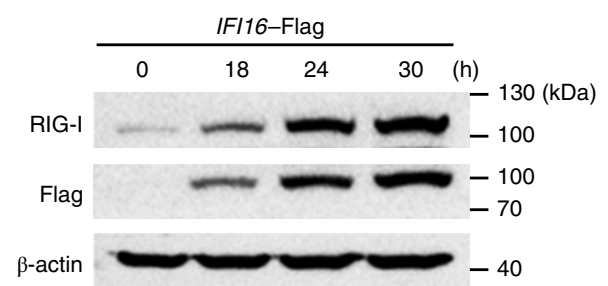

d

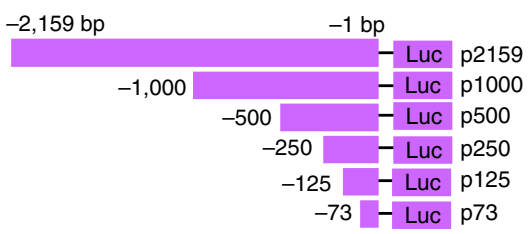

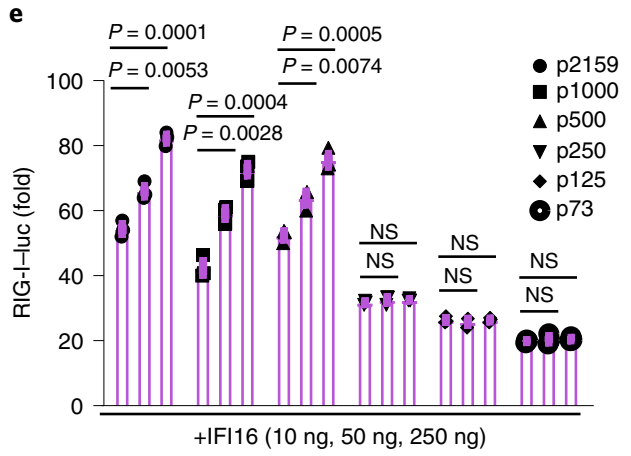

j

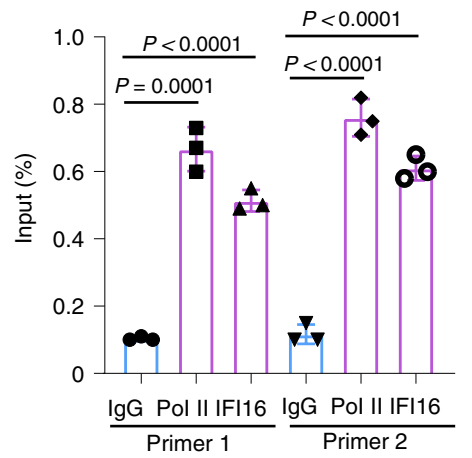

b

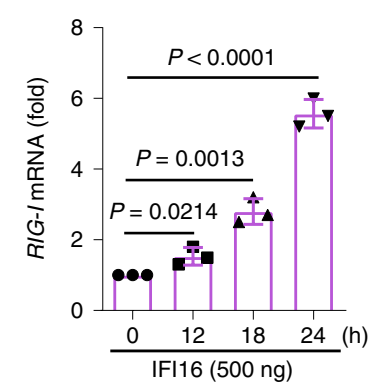

f

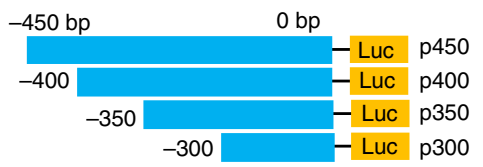

g

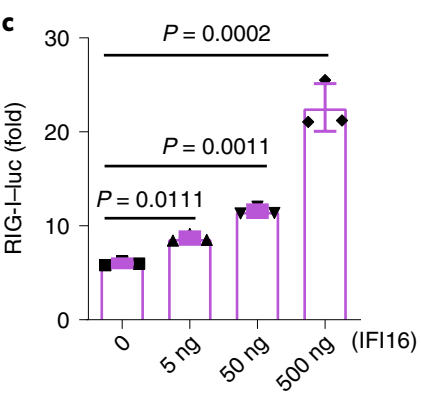

h

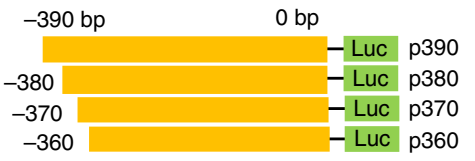

i

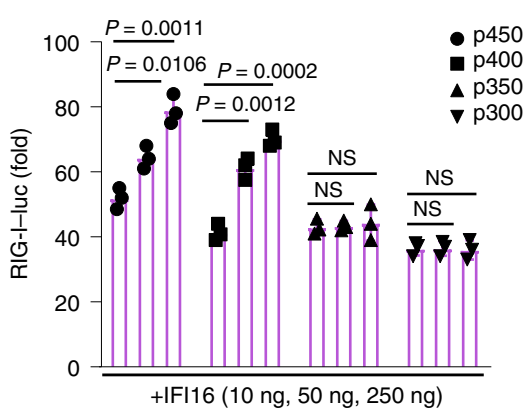

k

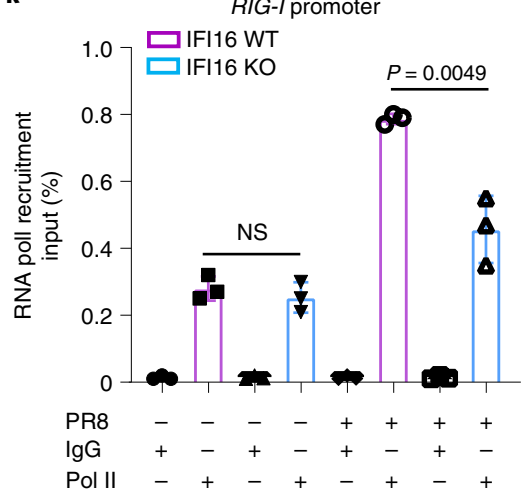

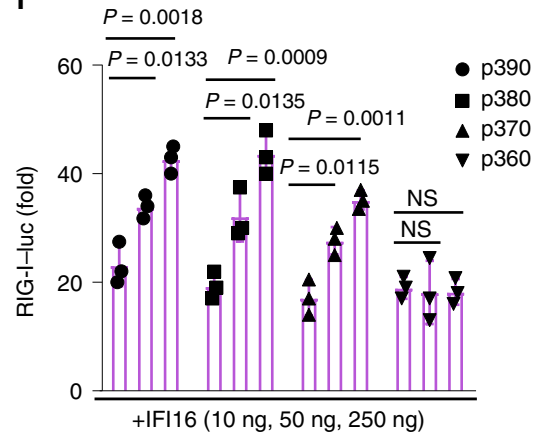

I

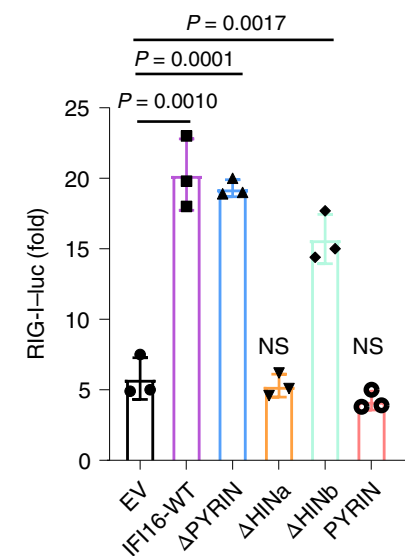

Fig. 5 | IFI16 upregulates RIG-I expression. a, RIG-I expression in IFl16-Flag overexpressing A549 cells at 0, 18, 24 and $30 \mathrm{~h}$ post transfection was detected by western blotting. $\beta$-actin detection was used as the loading control. b, RIG-I mRNA expression in A549 cells transfected with IFI16-Flag plasmids for 0, 12, 18 and $24 \mathrm{~h}$ was quantified by RT-qPCR. c Luciferase activity levels of the RIG-I reporter in HEK293 cells transfected with increasing amounts of IF/16-Flag expression vectors for $24 \mathrm{~h}$. Luciferase levels were normalized to Renilla levels. Values shown are fold changes over empty vector control. d, Schematic of the 2,000-bp promoter sequence of RIG-I and corresponding mutants. e, HEK293 cells were co-transfected with the RIG-I promoter mutant constructs and increasing amounts of IFI16-Flag as indicated. Luciferase levels were normalized to Renilla values. Values are shown as the fold-change over the empty vector control. $\mathbf{f}, \mathbf{g}$, Schematic of part of the RIG-I promoter sequence and every 50-bp deletion mutants (f), and a luciferase reporter assay ( $\mathbf{g}$ ) on the truncated mutants was performed as described in $\mathbf{e}$. h,i, Schematic of part of the RIG-I promoter sequence and every 10-bp deletion mutants (h), and a luciferase reporter assay (i) of these truncated mutants was performed as described in $\mathbf{e}$. $\mathbf{j}$, A549 cells were infected with PR8 virus for $12 \mathrm{~h}$ followed by ChIP assay. The promoter sequence binding to IFI16 was determined by RT-qPCR. k, IFI16 ${ }^{+/+}$and IFI16 ${ }^{-/-}$A549 cells were infected with PR8 virus for 0 and $12 \mathrm{~h}$, followed by ChIP assay. RNA Pol II recruitment to the RIG-I promoter was assessed by RT-qPCR. Data are presented as percent input minus IgG background. I, Luciferase activity of the RIG-I promoter-luciferase reporter in HEK293 cells transfected with full-length IFI16 vectors and truncated mutant plasmids. Luciferase levels were normalized to Renilla values. Values are shown as the fold-change over the empty vector control. For $\mathbf{a}$, data are representative of three independent experiments. For $\mathbf{b}-\mathbf{I}$, data are presented as the mean \pm s.d. from three independent experiments. Statistical significance in $\mathbf{b}, \mathbf{c}$ and $\mathbf{e}-\mathbf{I}$ was determined by unpaired two-tailed Student's $t$-test. 

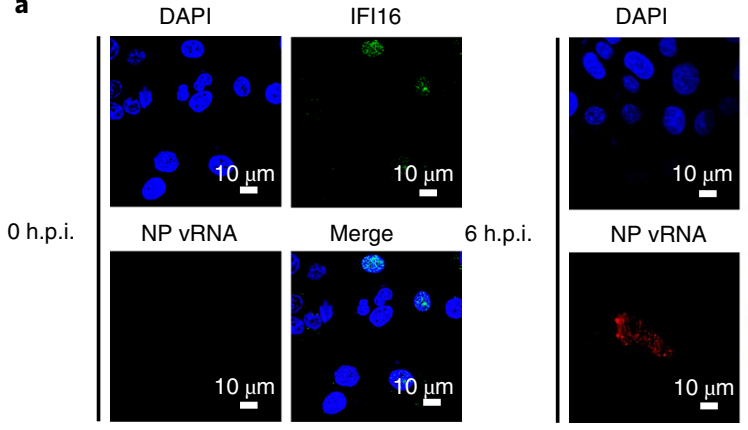
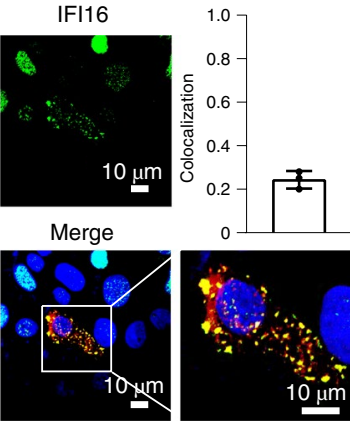

DAPI

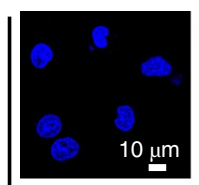

12 h.p.i.

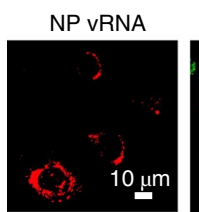

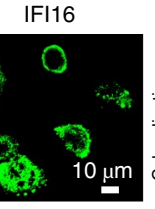

Merge

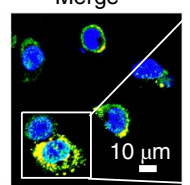

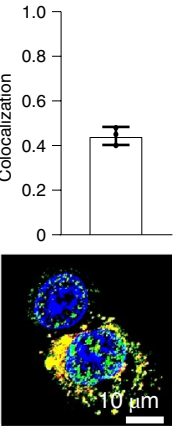

c

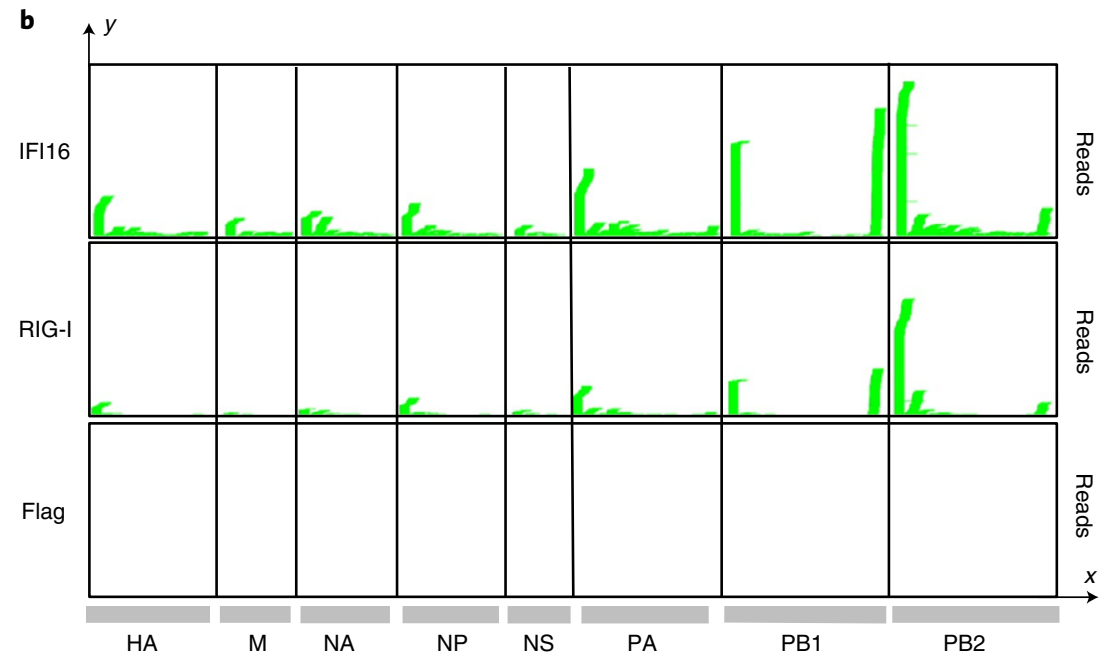

d

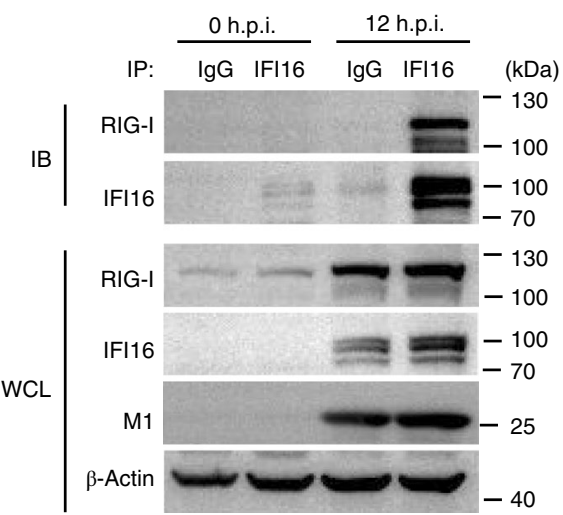

e

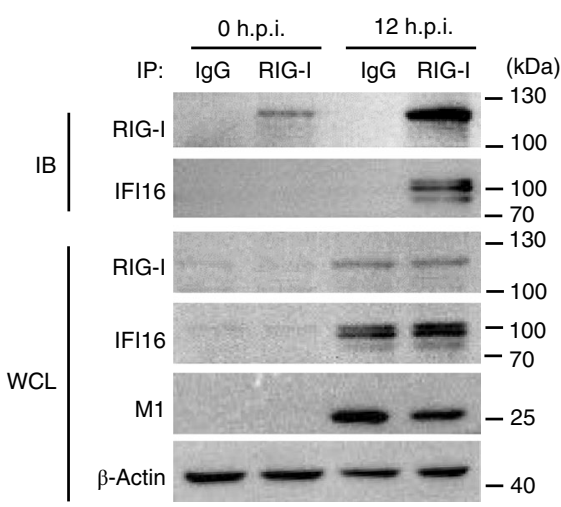

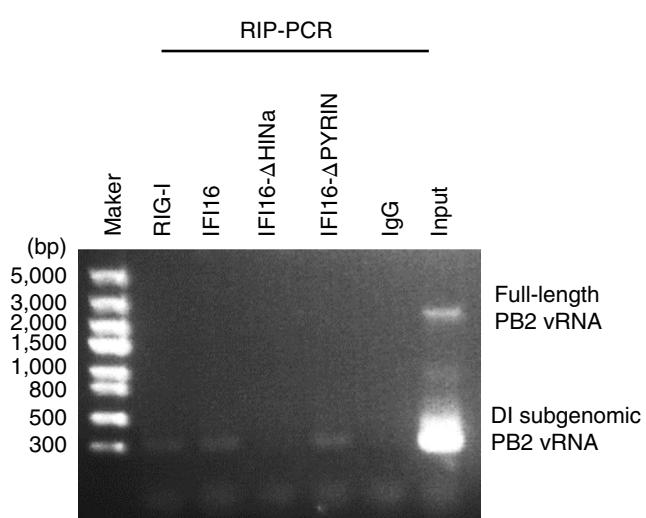

f PLA (IFI16 + RIG-I) + DAPI + M1 Enlarged

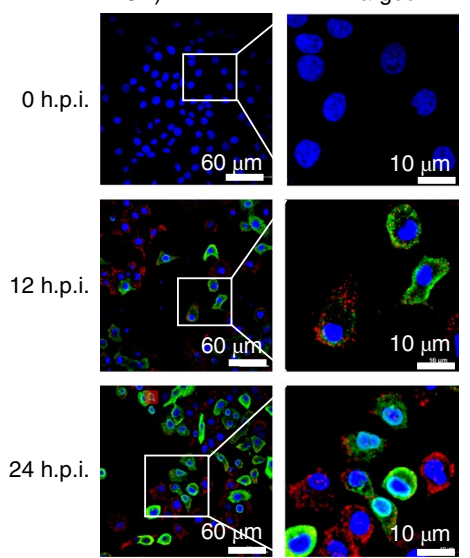

Fig. 6 | IFI16 binds vRNA and associates with RIG-I protein. a, Images showing colocalization of endogenous IFI16 (green) and viral NP RNA (red) in PR8-virus-infected A549 cells at 0,6 and 12 h.p.i. was detected by RNA FISH. Nuclei were stained with DAPI (blue). Insets: quantification of the colocalization of IFI16 and NP RNA in cells (bar plots). Data represent the mean \pm s.d. from three biological samples. b, Integrated Genome Viewer representation of captured IAV genomic (negative polarity) reads from Flag IPs of PR8-infected $R / G$ - $I^{-/-}$cells overexpressing IFI16-Flag (top) or RIG-I-Flag (bottom). Each horizontal green bar represents a single 150-nt read and the position where it aligns relative to an IAV gene segment. c, PCR detection of PB2 vRNAs in eluted RNA from RIG-I-Flag, IFI16-Flag and indicated Flag-tagged IFl16 deletions. d, A549 cells were infected with PR8 virus at 5 m.o.i. for the indicated duration, followed by co-IP with anti-IFI16 or IgG and immunoblotting analysis with anti-RIG-I antibody. e, A549 cells were infected with PR8 virus at 5 m.o.i. for the indicated duration, followed by co-IP with anti-RIG-I or IgG and immunoblotting analysis with anti-IFI16 antibody. f, A549 cells were infected with 1 m.o.i. of PR8 virus and then analysed by PLA with anti-IFI16 and anti-RIG-I antibodies at 0, 12 and 24 h.p.i. The right panels are enlarged. Red points indicate IFI16 plus RIG-I complexes, green points indicate the viral M1 protein. Data are representative of three independent experiments.

was also specifically enriched in genomic IAV segments during infection (Fig. 6b). Importantly, only WT IFI16 and IFI16 mutants bearing the HINa domain associated with vRNA fragments during infection (Fig. $6 \mathrm{c}$ and Extended Data Fig. 5d,e). Taken together, these results indicate that during infection, IFI16 can directly bind viral genome RNAs via its HINa domain.

To determine whether IFI16 is involved in the recognition of vRNAs by RIG-I during influenza virus infection, 
co-immunoprecipitation (co-IP) assays were performed, and the results demonstrated that there is an endogenous interaction of IFI16 and RIG-I during PR8 virus infection in A549 cells (Fig. 6d,e). Furthermore, in situ PLA microscopy showed colocalization of IFI16 and RIG-I in the cytoplasm of PR8-virus-infected A549 cells (Fig. 6f). Consistent with the PLA results, confocal microscopy also found an increasing interaction between IFI16 and RIG-I during IAV infection (Extended Data Fig. 5f). The PYRIN domain can mediate the interaction between IFI16 and host proteins ${ }^{38,39}$. Accordingly, the IFI16- $\Delta$ PYRIN-GFP mutant was unable to colocalize with RIG-I in HEK293 cells, thereby indicating that the PYRIN domain is required in the protein-protein interaction (Extended Data Fig. 5g). Interestingly, PYRIN-GFP transfection into HEK293 cells resulted in its protein colocalization with RIG-I, which formed filamentous structures (Extended Data Fig. 5h,i). IFI16 filamentous structures are involved in antiviral responses by associating with host restriction factors ${ }^{40-42}$. Collectively, these findings indicate that IFI16 binds influenza vRNAs and associates with RIG-I in influenza-virus-infected cells.

RIG-I is required for IFI16-mediated antiviral responses in IAV infection. K63-linked polyubiquitination of RIG-I by TRIM25 is essential for its activation ${ }^{11}$. To explore the potential role of IFI16 in the promotion of RIG-I signalling, we examined the interaction between TRIM25 and RIG-I in PR8-virus-infected IFI16 ${ }^{+/+}$ and $I F I 16^{-1-}$ A549 cells. Co-IP and in situ PLA experiments indicated that IFI16 deficiency impaired the interaction and colocalization between RIG-I and TRIM25 due to their reduced expression in infected IFI16 ${ }^{-/-}$cells, and inhibited K63-linked ployubiquitination of RIG-I during virus infection (Extended Data Figs. 6a,b and 7a). To further determine the impact of IFI16 on RIG-I polyubiquitination, we transfected HEK293 cells with Myc control or IFI16-Myc, RIG-I-Flag, TRIM25-His and either HA-ubiquitin, HA-ubiquitin-K48 or HA-ubiquitin-K63 plasmids. Co-IP experiments showed that IFI16 remarkably enhanced the interaction between TRIM25 and RIG-I and promoted RIG-I polyubiquitination in the presence of HA-ubiquitin and HA-ubiquitin-K63, but not HA-ubiquitin-K48 (Extended Data Fig. 7b). Thus, IFI16 facilitates TRIM25 binding to RIG-I and consequently promotes K63-linked polyubiquitination of RIG-I. RNA immunoprecipitation (RIP) and RT-qPCR assays showed that overexpression of IFI16 significantly enhanced the RIG-I binding to PA vRNA (Extended Data Fig. 7c). Correspondingly, IFI16 deficiency also markedly reduced RIG-I binding to PA vRNA in PR8-virus-infected A549 cells (Extended Data Fig. 7d). RIP and electrophoretic mobility shift assays (EMSAs) and RNA pull-down experiments further demonstrated that the binding of PA vRNA with RIG-I is sharply increased with increasing amounts of IFI16 (Extended Data Fig. 6c,d). Furthermore, RNA co-purified with RIG-I by RIP analysis from IAV-infected $I F I 16^{+/+}$ cells induced the production of IFN- $\beta$ more effectively than that from $I F I 16^{-1-}$ cells (Extended Data Fig. 7e,f), which suggests that IFI16 facilitates the stable binding of RIG-I to vRNAs during IAV infection. Importantly, only WT IFI16 and IFI16 mutants bearing the HINa and PYRIN domain, but not HINb, promoted vRNA binding to RIG-I (Extended Data Fig. 6e) and enhanced the production of IFN- $\beta$ during IAV infection (Extended Data Fig. 6f). Consistent with these observations, IFN- $\beta$ reporter assays showed that overexpression of IFI16 significantly enhanced PR8-virus-induced expression of IFN- $\beta$-luciferase reporter genes (Extended Data Fig. 7g). Moreover, the RIG-I-induced activity of IFN- $\beta$-luciferase reporter rose with increasing amounts of IFI16 expression vectors used in transfections (Extended Data Fig. 7h). Taken together, these results suggest that IFI16 promotes the activation of RIG-I signalling and, in so doing, boosts the production of IFN-I.

Finally, to investigate whether the antiviral function of IFI16 in IAV infection is dependent on RIG-I, A549 cells were transfected with the RIG-I- or MAVS-targeting siRNAs and then infected with IAV. Western blotting showed that the level of RIG-I or MAVS was significantly reduced by RIG-I-targeting siRNA-2835 (Extended Data Fig. 7i) or MAVS-targeting siRNA-571 transfection (Extended Data Fig. 6g). IAV infection could not effectively stimulate the production of IFN- $\beta$ in RIG-I- or MAVS-silenced IFI16 ${ }^{+/+}$A549 cells (Extended Data Figs. 6h and 7j). Overexpression of IFI16 in RIG-Ior MAVS-silenced A549 cells could not induce the production of IFN- $\beta$ during IAV infection (Extended Data Figs. 61 and $7 \mathrm{k}$ ). $R I G-I^{-1-}$ HEK293 cells were then transfected with IFI16-Flag or Flag vectors and then infected with PR8 virus at 1 m.o.i. Overexpression of IFI16 failed to inhibit viral NP and M1 replication in PR8-virus-infected $R I G-I^{-1-}$ cells at 6 and 12 h.p.i. (Extended Data Fig. 7l), whereas exogenous expression of RIG-I in $R I G-I^{-1-}$ cells rescued the inhibitory effect of IFI16 on viral titres at 24 h.p.i. (Extended Data Fig. $7 \mathrm{~m}$ ). Taken together, our results indicate that the antiviral function of IFI16 is dependent on RIG-I.

\section{Discussion}

Host cells possess critical sensors that can discriminate viral and host nucleic acids. IFI16 has been demonstrated to be a sensor of viral DNA in innate immune signalling ${ }^{16,17}$. However, its role in sensing vRNA during infection, in particular influenza virus infection, is hitherto unknown. In this study, we demonstrated that IFI16 is a viral RBP and is induced during influenza virus infection. IFI16 inhibited influenza virus replication in human cell lines and in mice through an enhanced induction of IFN-I. IFI16 enhances the transcription of RIG-I, binds vRNAs and interacts with the RIG-I protein, thereby increasing the sensitivity of RIG-I signalling. These findings establish a critical role for IFI16 in antiviral innate immune responses to influenza virus and possibly other RNA viruses (a proposed signalling model is depicted in Extended Data Fig. 8).

We found that IFI16 was strongly induced by IAV, which is consistent with previous findings that IFI16 can be induced by RNA virus infection and be upregulated by the type I and type III IFNs as an ISG ${ }^{27-29}$. IFI16 is known to boost sensing of intracellular DNA and subsequent IFN-I induction ${ }^{21,24,26}$; it functions as a cytoplasmic immune-sensor-mediating pyroptotic death of tissue $\mathrm{CD}^{+} \mathrm{T}$ cells abortively infected with HIV-1 (ref. ${ }^{22}$ ). Thus, the main role of IFI16 had been assumed to be a cytoplasmic sensor of viral DNA ${ }^{43}$. However, this is at odds with the findings that IFI16 is predominantly localized in the nucleus ${ }^{36}$. We found that IFI16 is induced in the nucleus and acts as a positive transcriptional regulator of $R I G-I$ during IAV infection. These results confirm previous findings that IFI16 regulates RNA-virus-mediated IFN-I responses as a transcription factor ${ }^{27}$. We also found that IFI16 was induced in the cytoplasm and sensed and interacted with influenza vRNAs, which is consistent with recent findings that IFI16 directly binds to incoming chikungunya virus genome RNA and acts as a pattern recognition receptor ${ }^{30}$. Overexpression of IFI16 in cells significantly increased IFN- $\beta$ expression and reduced viral titres, whereas knockdown or knockout of IFI16 had the opposite effects, which suggests that IFI16 is an important antiviral factor in IAV infection. Therefore, we demonstrated an important mechanism of IFI16 in RLR-mediated antiviral innate immune responses to influenza virus and furthered our understanding of the role of IFI16 in innate immunity.

The vRNA sensor RIG-I is thought to be the most important sensor of influenza virus infection ${ }^{9}$ in the recognition of cytoplasmic double-stranded RNA that leads to the transcriptional activation of IFN-I and downstream ISGs ${ }^{14}$. Thus, identification of positive regulators of RIG-I could be important in the control of virus infection. DNA-dependent activator of IFN-regulatory factor (DAI), a cytosolic DNA sensor, has already been found to recognize genomic RNA and regulate virus-induced cell-death pathways and thereby plays an important role in the pathogenesis of IAV infection ${ }^{44,45}$. 
Here, we demonstrated that IFI16 binds to vRNAs and enhances the RIG-I-mediated production of IFN-I during IAV infection. We further identified that IFI16 interacted with RIG-I via the PYRIN domain of IFI16. An interaction of IFI16 with RIG-I as a complex via siRNA was previously reported ${ }^{46}$. Notably, we found that IFI16 facilitates RIG-I binding to IAV vRNAs during infection and promotes virus-induced K63-linked polyubiquitination of RIG-I, thereby indicating that IFI16 positively contributes to RIG-I-dependent antiviral responses. It is currently not clear whether the filamentous structures of IFI16 are necessary for IFI16-vRNA and IFI16-RIG-I interactions or about the types of vRNA structures that are needed to interact with IFI16.

Collectively, our study showed that IFI16 induced by influenza virus infection positively regulates RIG-I signalling by enhancing its transcriptional expression through recruitment of RNA Pol II to the RIG-I promoter, and sensing of vRNA to promote virus-induced K63-linked polyubiquitination of RIG-I. This study highlights an important mechanism of IFI16 in RLR-mediated innate antiviral immune responses to IAV and possibly other RNA virus infections, and expands our understanding of the functions of the innate immune system in intracellular virus recognition, which could help the development of new strategies to modulate antiviral responses.

\section{Methods}

Ethics statement. All animal experiments were performed in accordance with institutional guidelines of China Agricultural University (CAU) (approval SKLAB-B-2010-003) and approved by the Beijing Association for Science and Technology of China (approval SYXK, Beijing, 2007-0023).

Cells. Madin-Darby canine kidney cells (MDCK), human embryonic kidney cells (HEK293) and human lung adenocarcinoma epithelial cells (A549) were maintained in-house. The THP-1 cell line was kindly provided by S. Zheng (China Agricultural University). IFI16 ${ }^{-/-}$A549 and RIG-I $I^{-1-}$ HEK293 cell lines were generously given by Y. Chen (Sun Yat-sen University) and W. Liu (Institute of Microbiology, Chinese Academy of Sciences), respectively. The IFNAR1 $1^{-/-}$A549 cell line was generously given by Y. Zhu (Wuhan University), and the 2fTGH-ISRE cell line was kindly provided by F. You (Peking University) ${ }^{47}$. HEK293T-Gluc cells were kindly provided by S. Cen (Chinese Academy of Medical Sciences and Peking Union Medical School $)^{48}$. Primary BMDMs were produced as previously described ${ }^{49}$. Cells were cultured in DMEM supplemented with $10 \%(\mathrm{v} / \mathrm{v})$ heat-inactivated fetal bovine serum (Gibco), $100 \mathrm{U} \mathrm{ml}^{-1}$ penicillin and $100 \mu \mathrm{g} \mathrm{ml}^{-1}$ streptomycin.

Viruses. The influenza A/Puerto Rico/8/1934 (PR8; H1N1) strain was maintained in our laboratory. The PR8-GFP virus was generated by inserting the GFP CDS sequences at the carboxy terminal of NS1 as previously described ${ }^{50}$. The methods of cell culture, mice infection and virus titration in lung tissues were performed as previously described ${ }^{49}$.

Plasmid construction and transfection. IFI16, RIG-I, TBK1, TRIM25 and MAVS genes were amplified by PCR using PR8-virus-infected THP-1 cells at 8 h.p.i. Full-length and mutated IFI16 expression constructs were generated using PRK5 containing different tags or pCDNA3.1-GFP vectors by recombinase-mediated recombination. Full-length RIG-I expression vectors were created using PRK5-Flag or pCDNA3.1-mCherry vectors. Full promoter sequences or truncations of RIG-I were generated using a PGL3.0 luciferase reporter (Promega). Plasmid transfection experiments in HEK293 or A549 cells were performed using Lipofectamine 3000 reagent (Invitrogen). IFI16 $6^{+/+}$or IFI16 $6^{-/-}$A549 cells were transfected with the indicated siRNAs using Lipofectamine RNAiMAX reagent (Invitrogen).

In vivo virus infection. $p 204^{-/-} \mathrm{C} 57 \mathrm{BL} / 6$ mice were kindly provided by $\mathrm{W}$. Tang (Shandong University). Sex- and age-matched WT C57BL/6 mice were purchased from Laboratory Animal Technology of Charles River, Beijing. Seven-week-old mice were intranasally inoculated with PR8 virus at a 50\% tissue culture infectious dose $\left(\mathrm{TCID}_{50}\right)$ of 50 in $50 \mu \mathrm{l}$ of PBS. Body weight and survival were monitored daily after infection. Lung tissue lysates were generated by homogenizing snap-frozen lung tissues two times ( $20 \mathrm{~s}$ each time) in MEM medium and centrifuging the lung suspensions at 2,000 r.p.m. for $15 \mathrm{~min}$. TCID 50 assays were performed using MDCK cells and TCID T0 $_{0}$ values were calculated as previously described ${ }^{49}$. WT and $p 204^{-1-}$ mice were intranasally innoculated with PR8 virus at a dose $100 \mathrm{TCID}_{50}$. BALF was collected at 0 and 3 d.p.i. A BD CBA Mouse Inflammation kit (BD Biosciences, 552364) was used to quantitatively measure IL-6 and MCP-1 levels in BALF.

Antibodies and reagents. Anti-IFI16 (ab185812), anti-TBK1 (ab40676), anti-NP (ab104870), anti-ubiquitin (linkage-specific K63) (ab271929) and anti-RNA Pol II (ab238450) antibodies were from Abcam. Anti-IFI16 (sc-8023) and anti-TRIM25 (sc-166926) antibodies were from Santa Cruz. Rabbit anti-p65 (10745-1-AP), anti-HA (66006-2-Ig) and anti-RIG-I (20566-1-AP) antibodies were from Proteintech. Anti-IRF3 (YT2398) antibody was from ImmunoWay Biotechnology Company. Anti-phospho-TBK1 (Ser172; 5483); anti-phospho-IRF3 (Ser396; 4947), anti-IFI16 (14970S), anti-FLAG (8146), anti-His (9991) anti-Myc (2278) and anti-phospho-p65 (Ser536; 3033) antibodies were from Cell Signaling Technology. Poly(I:C) (TLRL-PIC) was from InvivoGen. Recombinant human IFN- $\gamma$ (HY-P7025) and C-646 (HY-13823, $10 \mathrm{mg}$ ) was from MedChemExpress.

In vitro transcription and biotin-labelling RNA purification. Templates for T7 RNA transcription were linearized from H7N9 pSPT9 plasmids coding for individual RNA segments of H7N9 virus. T7 transcription reactions were carried out with T7 RNA polymerase in transcription buffer and biotin-dNTPs mix according to the manufacturer's instructions (Promega) ${ }^{51}$. Following DNase I treatment, biotin-labelled vRNAs were extracted with phenol-chloroform, ethanol precipitated and purified with RNAeasy columns (Aidlab Biotechnologies) and analysed on denaturing agarose gels for correct size.

AP-MS. THP-1 cells were treated with phorbolmyristateacetate for $12 \mathrm{~h}$, and infected with H7N9 virus (1 m.o.i.). After $12 \mathrm{~h}$ of infection, cells were lysed with lysis buffer and incubated with in vitro-transcribed 2 pM biotin-labelled vRNAs for $4 \mathrm{~h}$ followed by incubation with pre-washed Dynabeads M-280 streptavidin (Sigma) for $3 \mathrm{~h}$ at $4{ }^{\circ} \mathrm{C}$. Beads were washed five times with NT2 buffer (50 mM Tris- $\mathrm{HCl}$ pH 7.0, $150 \mathrm{mM} \mathrm{NaCl}_{1} 1 \mathrm{mM} \mathrm{MgCl}_{2}$ and $0.05 \% \mathrm{NP}-40$ ). Protein complex bound to the beads was then boiled with $20 \mu \mathrm{PBS}$ at $100^{\circ} \mathrm{C}$ for $10 \mathrm{~min}$. Pulled-down proteins were identified by liquid chromatography-MS.

Western blotting. Western blotting was performed as previously described ${ }^{49}$. Briefly, protein samples were mixed with loading buffer supplemented with $10 \% \beta$-mercaptoethanol, heated at $95^{\circ} \mathrm{C}$ for $5 \mathrm{~min}$ and separated on a $10 \%$ SDSPAGE under reducing conditions. After electrophoresis, protein samples were electroblotted onto polyvinylidene difluoride membranes (Bio-Rad) and blocked for $2 \mathrm{~h}$ in Tris-buffered saline $(10 \mathrm{mM}$ Tris- $\mathrm{HCl}, \mathrm{pH} 8.0$, containing $150 \mathrm{mM} \mathrm{NaCl})$ containing $5 \%(\mathrm{w} / \mathrm{v})$ non-fat dry milk and $0.5 \%$ (v/v) Tween-20. The blots were incubated with the primary antibodies overnight at $4{ }^{\circ} \mathrm{C}$. The next day, the blots were incubated with corresponding horseradish peroxidase (HRP)-conjugated secondary antibodies for $1 \mathrm{~h}$ at room temperature (RT). HRP antibody binding was detected using a standard enhanced chemiluminescence (ECL) kit (Thermo Scientific).

RT-qPCR. Total RNA from virus-infected cells or lung tissues was extracted using an RNA isolation kit (Thermo Scientific). First-strand complementary DNA was synthesized from $1 \mu \mathrm{g}$ of total RNA using a TransScript RT reagent kit (TransGen). Uni-12 primer was used for the detection of influenza vRNA, and oligo dT and random primers were used for detecting host and viral genes. Generated cDNA was subjected to qPCR in a $25-\mu \mathrm{l}$ reaction volume using FastStart Universal SYBR Green master mix (Roche). Human $\beta$-actin (ACTB) and mouse GAPDH genes were amplified for normalization of the cDNA amount used in qPCR. Reactions were conducted in triplicate, and the data were analysed using the $2^{-\Delta \Delta \mathrm{Ct}}$ method. qPCR primers used in this study are provided in the Supplementary Information.

IFN-I bioassay. During PR8 virus infection, IFN-I released in the human cell culture medium was quantified in 2 TTGH-ISRE cells as previously described ${ }^{52}$. In brief, $200 \mathrm{ml}$ of cell culture supernatant was incubated with confluent $2 \mathrm{fGTH}$-ISRE-Luci cells in a 24 -well plate for $6 \mathrm{~h}$. Cells were then lysed in passive lysis buffer and subjected to luciferase quantification (Promega). A serial dilution of recombinant human IFN- $\beta$ (Invitrogen) was used as reference.

Luciferase reporter assays. HEK293 cells seeded on 24-well plates were co-transfected with $125 \mathrm{ng}$ of the luciferase reporter plasmids and an equal amount of various expression plasmids or empty controls. Ten nanograms of pRL-TK plasmid was used as an internal control. At $24 \mathrm{~h}$ after transfection, Renilla luciferase and firefly luciferase expression levels were measured in the presence or absence of virus stimulation for $12 \mathrm{~h}$ using a Dual-Luciferase Reporter Assay kit (Promega) in a TD-20/20 Luminometer according to the manufacturers' recommendations. Results were normalized to corresponding control reporter constructs.

Gaussia luciferase reporter assays were performed according to standard procedures described in detail elsewhere ${ }^{48}$. Briefly, HEK293T-Gluc cells were transfected with either IFI16-Flag or Flag control plasmids followed by infection with PR8 virus ( 1 m.o.i.) for $24 \mathrm{~h}$, and viral infectivity was evaluated by measuring Gluc activity. After a further $24 \mathrm{~h}$ of incubation, cell supernatants were collected and measured for Gluc activity. Dimethylsulfoxide was used as negative controls. The inhibition rate of the tested compounds was calculated with the following equation, where RLU indicates relative light unit:

$$
\text { Inhibition rate }=\left(\mathrm{RLU}_{\text {infected cells }}-\mathrm{RLU}_{\text {tested compound }}\right) /
$$

$$
\left(\mathrm{RLU}_{\text {infected cells }}-\mathrm{RL} \mathrm{U}_{\text {mock-infected cells }}\right) 100 \%
$$


Histology. WT and $p 204^{-1-}$ mice were euthanized at the indicated time points after PR8 virus infection. Lung tissues were collected and fixed with $4 \%$ formaldehyde, followed by paraffin embedding. For histopathological analysis, 5 - to $7-\mu \mathrm{m}$ sections were sectioned longitudinally through the left and right lung and stained using a standard haematoxylin and eosin (H\&E) protocol.

Immunohistochemistry. Lung sections were deparaffinized with xylene and rehydrated with ethanol gradations and water. Endogenous peroxidase activity was blocked using $3 \%$ hydrogen peroxide in methanol. PBS containing $0.05 \%$ Tween- 20 was used to wash lung tissue sections between steps. Lung sections were incubated with the primary anti-NP antibody (ab20343) at 1:100 dilution or with the isotype control at the same concentration at $4{ }^{\circ} \mathrm{C}$ overnight in a humidified chamber. Sections were subsequently incubated with the HRP-conjugated secondary antibody for $60 \mathrm{~min}$ at RT. Immunodetection was performed using a Vector Elite ABC kit (Vectastain, Vector).

Co-IP. For the co-IP assays, A549 cells were infected with PR8 virus (5 m.o.i.). After infection, cell samples were collected and lysed in $800 \mu \mathrm{l}$ of IP lysis buffer (Thermo Scientific) containing protease and phosphatase inhibitors. A portion of each whole cell lysate sample was kept to confirm protein expression levels, and $500 \mu \mathrm{g}$ of cell lysate was used for the co-IP assays. Lysates were incubated with anti-IFI16, anti-RIG-I or IgG antibodies overnight at $4{ }^{\circ} \mathrm{C}$ under constant rotation, and then $40 \mu \mathrm{l}$ of protein $\mathrm{A} / \mathrm{G}$ beads (Santa Cruz) were added and incubated for $2 \mathrm{~h}$ at $4{ }^{\circ} \mathrm{C}$ under gentle rotation. The beads were then washed four times with cold lysis buffer and analysed by SDS-PAGE and western blotting.

RIP-qPCR. Two $10-\mathrm{cm}^{2}$ dishes $\left(10^{7}\right.$ cells per dish) of A549 cells were infected with PR8 virus for $12 \mathrm{~h}$. Cells were lysed with RIP lysis buffer (50 mM HEPES, $150 \mathrm{mM} \mathrm{KCl}, 2 \mathrm{mM}$ EDTA, $1 \mathrm{mM} \mathrm{NaF}, 0.5 \% \mathrm{NP} 40,0.5 \mathrm{mM}$ dithiothreitol (DTT), $1 \times$ protease inhibitor cocktail and $25 \mathrm{U}$ RNasin) for $30 \mathrm{~min}$ at $4^{\circ} \mathrm{C}$. Cell lysates were centrifuged at 12,000 r.p.m. for $15 \mathrm{~min}$ at $4{ }^{\circ} \mathrm{C}$ and the supernatants were subjected to RIP. A $50 \mu \mathrm{l}$ aliquot of cell supernatant was saved as input, and the remaining samples were each incubated with $5 \mu$ g anti-IFI16 antibody or IgG antibody and $40 \mu \mathrm{l}$ protein $\mathrm{A} / \mathrm{G}$ beads for $10 \mathrm{~h}$ at $4^{\circ} \mathrm{C}$ under gentle shaking. After IP, the beads were pelleted and washed four times with RIP wash buffer ( $50 \mathrm{mM}$ Tris $\mathrm{pH} 7.4$, $150 \mathrm{mM} \mathrm{NaCl}, 1 \mathrm{mM} \mathrm{MgCl}{ }_{2}$ and $0.05 \% \mathrm{NP} 40$ ), resuspended in $250 \mathrm{ml}$ of DNase digestion buffer (40 $\mathrm{mM}$ Tris pH 8.0, $10 \mathrm{mM} \mathrm{MgSO}_{4}$ and $1 \mathrm{mM} \mathrm{CaCl}_{2}$ ) and treated with $25 \mathrm{U}$ RNasin (Promega) and $2 \mathrm{U}$ DNase I (NEB) at $37^{\circ} \mathrm{C}$ for $20 \mathrm{~min}$. Beads were then washed and resuspended in $100 \mu$ RIP wash buffer, $10 \%$ of each sample was removed for immunoblot analysis. Samples were treated with $4 \mathrm{U}$ proteinase $\mathrm{K}$ at $55^{\circ} \mathrm{C}$ for $30 \mathrm{~min}$. The input and immunoprecipitated RNAs were isolated by $1 \mathrm{ml}$ of TRIzol reagent (Sigma), and vRNAs were analysed by RT-qPCR or RNA sequencing.

ChIP-qPCR. Approximately $10^{7}$ treated cells were cross-linked with $1 \%$ formaldehyde at RT for $10 \mathrm{~min}$, and the crosslinking was quenched with $0.125 \mathrm{M}$ glycine for $5 \mathrm{~min}$. Cells were then collected by centrifugation, lysed with SDS lysis buffer containing protease inhibitor cocktail and sonicated to shear the DNA. The sonicated DNA-protein complexes were incubated with anti-IFI16 (Cell Signaling Technology, D8B5T), anti-RNA Pol II (Abcam, ab238450) or control IgG (Beyotime, A7016) antibodies. The immunocomplexes were collected using Protein G Dynabeads (10004d, Invitrogen). The Dynabeads were washed once with wash buffer A (20 mM Tris-HCl ( $\mathrm{pH} 8.0), 500 \mathrm{mM} \mathrm{NaCl}, 2 \mathrm{mM}$ EDTA, $0.1 \%$ SDS and $1 \%$ Triton X-100), once with wash buffer B (10 mM Tris- $\mathrm{HCl}$ (pH 8.0), $250 \mathrm{mM}$ $\mathrm{LiCl}, 1 \mathrm{mM}$ EDTA and $1 \% \mathrm{NP}-40)$ and three times with wash buffer $\mathrm{C}(1 \mathrm{mM}$ EDTA, $10 \mathrm{mM}$ Tris- $\mathrm{HCl}, \mathrm{pH} 8.0$ ). The beads were eluted with $100 \mathrm{ml}$ elution buffer $(50 \mathrm{mM}$ Tris- $\mathrm{HCl}$ (pH 8.0), $10 \mathrm{mM}$ EDTA and 1\% SDS) followed by incubation at $65^{\circ} \mathrm{C}$ overnight to reverse crosslinking. The next day, the DNA was purified with a QIAquick PCR purification kit (Magen, D211102) and analysed using FastStart Universal SYBR Green master mix (Roche).

Confocal microscopy. A549 or HEK293 cells on coverslips were washed twice with pre-warmed PBS and fixed with $4 \%$ paraformaldehyde for $15 \mathrm{~min}$ at RT. Cells were subsequently permeabilized with immunostaining permeabilization buffer containing Triton X-100 (Beyotime) for $10 \mathrm{~min}$ and blocked in QuickBlock blocking buffer for $20 \mathrm{~min}$ at RT. Fixed cells were incubated with indicated antibodies diluted in immunostaining primary antibody dilution buffer at $4^{\circ} \mathrm{C}$ overnight. Coverslips were then washed three times with PBS and incubated with Alexa Fluor 488-conjugated secondary antibodies or Alexa Fluor 555-conjugated secondary antibodies for $1 \mathrm{~h}$ at $37^{\circ} \mathrm{C}$. Coverslips were finally washed three times and mounted onto microscope slides with 4,6-diamidino-2-phenylindole (DAPI) staining solution (Beyotime) for $8 \mathrm{~min}$ and examined by confocal microscopy. Immunostained cells were visualized using a Nikon super-resolution laser scanning confocal microscope under a $\times 100$ oil objective and analysed using the Imaris 9.2 platform.

In situ PLA microscopy. A DuoLink PLA kit (DUO92105-1KT, Sigma) was used to test protein-protein interactions as described in the protocol. WT or IFI16 ${ }^{-1-}$ A549 cells were infected with PR8 virus at 1 m.o.i. for $12 \mathrm{~h}$, fixed and permeabilized as described in the "Confocal microscopy" section and blocked with DuoLink blocking buffer for $30 \mathrm{~min}$ at $37^{\circ} \mathrm{C}$. Cells were incubated with corresponding primary antibodies diluted in DuoLink dilution buffer. After washing, cells were incubated with species-specific PLA probes (Plus and Minus) for $1 \mathrm{~h}$ at $37^{\circ} \mathrm{C}$ under hybridization conditions and in the presence of two additional oligonucleotides to facilitate hybridization of PLA probes if they were in close proximity $(<40 \mathrm{~nm})$. Ligase was then added and incubated for $30 \mathrm{~min}$ at $37^{\circ} \mathrm{C}$ to join hybridized oligonucleotides. Amplification polymerase was added to generate a concatemeric product extending from the oligonucleotide arm of the PLA probe. Finally, a detection solution consisting of fluorescently labelled oligonucleotides was added, and the labelled oligonucleotides were hybridized to the concatemeric products. Nuclei was stained with Duolink in situ mounting medium containing DAPI.

Ubiquitination assay. HEK293 cells were transfected with plasmids encoding RIG-I-Flag, TRIM25-His with or without coexpression of IFI16-Myc or Myc empty vectors, and HA-ubiquitin (WT) or HA-ubiquitin mutants (K48 or K63). After $24 \mathrm{~h}$ of transfection, cells were collected and lysed in RIPA buffer $(50 \mathrm{mM}$ Tris- $\mathrm{HCl}$ (pH 8.0), $150 \mathrm{mM} \mathrm{NaCl}, 1 \% \mathrm{NP}-40,0.1 \%$ SDS and 1 mM EDTA) containing protease inhibitor cocktail and $10 \mu \mathrm{M}$ deubiquitinase inhibitor $\mathrm{N}$-ethylmaleimide (Sigma). The cell extracts were immunoprecipitated with anti-Flag antibody overnight at $4^{\circ} \mathrm{C}$ and then beads were added to the samples for $1-1.5 \mathrm{~h}$ at $4^{\circ} \mathrm{C}$. The beads were washed three times with RIPA buffer and analysed by immunoblotting with anti-HA antibody.

RNA FISH. A549 cells were grown in 24-well slide chambers and infected with PR8 at a m.o.i. of 1 . At 12 h.p.i., cells were fixed for $15 \mathrm{~min}$ in $4 \%$ paraformaldehyde, then permeabilized and dehydrated by sequential 3-min incubations as follows: once with $70 \%$ ethanol, once with $85 \%$ ethanol and three times with $100 \%$ ethanol. Cells were then hybridized with the Alexa Fluor 488-conjugated NP RNA target probes (NP-probes, GenePharma) of PR8 virus in hybridization buffer for $10 \mathrm{~min}$ at $75^{\circ} \mathrm{C}$. Cells were further incubated for $12-16 \mathrm{~h}$ at $37^{\circ} \mathrm{C}$. Finally, cells were stained with anti-IFI16 and secondary antibodies, and nuclei were stained with DAPI as previously described.

MST. HEK293 cells were separately transfected with the HINa-GFP, HINb-GFP and PYRIN-GFP expression vectors. After $24 \mathrm{~h}$ of transfection, cell lysates were collected and incubated with twofold serial dilutions of indicated vRNAs in MST-optimized buffer at a constant concentration $(20-100 \mathrm{nM})$. Equal volumes of binding reactions were mixed by pipetting and incubated for $15 \mathrm{~min}$ at RT. Mixtures were enclosed in standard-treated glass capillaries and loaded into the instrument (Monolith NT.115, NanoTemper). To identify whether IFI16 directly binds to the IAV RNAs, the transcribed RNAs were labelled with fluorescein RNA labelling mix (11685619910, Sigma) and purified as previously described ${ }^{53}$. Purified GST-IFI16 proteins (Abcam, ab158724) were incubated with different amounts of IAV full-length fluorescein-labelled RNAs, followed by MST assays. For all the measurements, 200-1,000 counts were obtained for the fluorescence intensity. Dissociation constant $\left(K_{\mathrm{d}}\right)$ values were determined using the NanoTemper analysis tool.

RNA pull-down assay. HEK293 cells were transfected with Flag-tagged RIG-I, IFI16, TBK1 or MAVS vectors. After $24 \mathrm{~h}$ of transfection, cells were collected and lysed with lysis buffer (50 mM Tris- $\mathrm{HCl} \mathrm{pH} \mathrm{7.0,} 150 \mathrm{mM} \mathrm{NaCl}, 1 \mathrm{mM} \mathrm{MgCl}$ and $0.05 \%$ NP-40). Cell lysates were mixed with transcribed biotin-labelled viral NP RNA for $4 \mathrm{~h}$ at $4^{\circ} \mathrm{C}$ and incubated with pre-washed Dynabeads M-280 Streptavidin (Sigma) for another $3 \mathrm{~h}$ at $4{ }^{\circ} \mathrm{C}$. The protein samples bound to the beads were boiled and analysed by SDS-PAGE and western blotting.

DNA pull-down assay. IFI16 proteins were purified from IFI16Flag-overexpressing HEK293 cells by immunoprecipitation using M2 beads (Sigma). Biotinylated mutant DNA probes were synthesized using an EMSA Probe Biotin Labelling kit (Beyotime, GS008) and were annealed and incubated with the purified Flag-tagged IFI16 proteins for $30 \mathrm{~min}$ in binding buffer $(10 \mathrm{mM}$ Tris, $1 \mathrm{mM} \mathrm{KCl}, 1 \% \mathrm{NP}-40,1 \mathrm{mM}$ EDTA and 5\% glycerol) at RT. Then, $40 \mu \mathrm{l}$ prewashed Dynabeads M-280 Streptavidin (Sigma) was added for incubation at $4{ }^{\circ} \mathrm{C}$ for $1 \mathrm{~h}$. The mutant probe-binding proteins were eluted by boiling and analysed by immunoblotting.

Statistical analysis. For all the bar graphs, data are shown as the mean \pm s.e.m. All statistical analyses were performed using GraphPad Prism software v.7.00 (GraphPad Software). The Kaplan-Meier method was employed for survival analysis. Differences in means were considered statistically significant at $P<0.05$. And significance levels are as follow: ${ }^{\star} P<0.05 ;{ }^{\star *} P<0.01$; ${ }^{\star *} P<0.001$; NS, not significant.

Reporting Summary. Further information on research design is available in the Nature Research Reporting Summary linked to this article.

\section{Data availability}

The MS proteomics data have been deposited with the ProteomeXchange Consortium via the $\mathrm{PRIDE}^{54}$ partner repository (https://www.ebi.ac.uk/pride/) with the dataset identifiers PXD020723 and PXD020723. The accession numbers 
for the RNA sequencing data are GSE157609 and GSE158122. Source data are provided with this paper.

Received: 23 April 2020; Accepted: 6 April 2021;

Published online: 13 May 2021

\section{References}

1. Collins, P. J. et al. Crystal structures of oseltamivir-resistant influenza virus neuraminidase mutants. Nature 453, 1258-1261 (2008).

2. Wang, M. Z., Tai, C. Y. \& Mendel, D. B. Mechanism by which mutations at His274 alter sensitivity of influenza A virus N1 neuraminidase to oseltamivir carboxylate and zanamivir. Antimicrob. Agents Chemother. 46, 3809-3816 (2002).

3. Deyde, V. M. et al. Surveillance of resistance to adamantanes among influenza $\mathrm{A}(\mathrm{H} 3 \mathrm{~N} 2)$ and $\mathrm{A}(\mathrm{H} 1 \mathrm{~N} 1)$ viruses isolated worldwide. J. Infect. Dis. 196, 249-257 (2007).

4. Furuse, Y., Suzuki, A. \& Oshitani, H. Large-scale sequence analysis of M gene of influenza A viruses from different species: mechanisms for emergence and spread of amantadine resistance. Antimicrob. Agents Chemother. 53, 4457-4463 (2009)

5. Eisfeld, A. J., Neumann, G. \& Kawaoka, Y. At the centre: influenza A virus ribonucleoproteins. Nat. Rev. Microbiol. 13, 28-41 (2015).

6. Blasius, A. L. \& Beutler, B. Intracellular Toll-like receptors. Immunity 32, 305-315 (2010).

7. Takeuchi, O. \& Akira, S. Innate immunity to virus infection. Immunol. Rev 227, 75-86 (2009)

8. Pang, I. K. \& Iwasaki, A. Inflammasomes as mediators of immunity against influenza virus. Trends Immunol. 32, 34-41 (2011).

9. Kato, H. et al. Differential roles of MDA5 and RIG-I helicases in the recognition of RNA viruses. Nature 441, 101-105 (2006).

10. Pichlmair, A. et al. RIG-I-mediated antiviral responses to single-stranded RNA bearing 5'-phosphates. Science 314, 997-1001 (2006).

11. Gack, M. U. et al. TRIM25 RING-finger E3 ubiquitin ligase is essential for RIG-I-mediated antiviral activity. Nature 446, 916-920 (2007).

12. Oshiumi, H. et al. The ubiquitin ligase Riplet is essential for RIG-I-dependent innate immune responses to RNA virus infection. Cell Host Microbe 8, 496-509 (2010).

13. Jiang, X. et al. Ubiquitin-induced oligomerization of the RNA sensors RIG-I and MDA5 activates antiviral innate immune response. Immunity $\mathbf{3 6}$, 959-973 (2012).

14. Loo, Y. M. \& Gale, M. Jr. Immune signaling by RIG-I-like receptors. Immunity 34, 680-692 (2011).

15. Dawson, M. J. \& Trapani, J. A. The interferon-inducible autoantigen, IFI 16 localization to the nucleolus and identification of a DNA-binding domain. Biochem. Biophys. Res. Commun. 214, 152-162 (1995).

16. Orzalli, M. H., Conwell, S. E., Berrios, C., DeCaprio, J. A. \& Knipe, D. M. Nuclear interferon-inducible protein 16 promotes silencing of herpesviral and transfected DNA. Proc. Natl Acad. Sci. USA 110, E4492-E4501 (2013).

17. Johnson, K. E. et al. IFI16 restricts HSV-1 replication by accumulating on the hsv-1 genome, repressing HSV-1 gene expression, and directly or indirectly modulating histone modifications. PLoS Pathog. 10, e1004503 (2014).

18. Almine, J. F. et al. IFI16 and cGAS cooperate in the activation of STING during DNA sensing in human keratinocytes. Nat. Commun. 8, 14392 (2017).

19. Goubau, D., Deddouche, S. \& Reis e Sousa, C. Cytosolic sensing of viruses. Immunity 38, 855-869 (2013).

20. Jonsson, K. L. et al. IFI16 is required for DNA sensing in human macrophages by promoting production and function of cGAMP. Nat. Commun. 8, 14391 (2017).

21. Kerur, N. et al. IFI16 acts as a nuclear pathogen sensor to induce the inflammasome in response to Kaposi sarcoma-associated herpesvirus infection. Cell Host Microbe 9, 363-375 (2011).

22. Monroe, K. M. et al. IFI16 DNA sensor is required for death of lymphoid CD4 T cells abortively infected with HIV. Science 343, 428-432 (2014).

23. Orzalli, M. H. et al. cGAS-mediated stabilization of IFI16 promotes innate signaling during herpes simplex virus infection. Proc. Natl Acad. Sci. USA 112, E1773-E1781 (2015).

24. Unterholzner, L. et al. IFI16 is an innate immune sensor for intracellular DNA. Nat. Immunol. 11, 997-1004 (2010).

25. Ansari, M. A. et al. Herpesvirus genome recognition induced acetylation of nuclear IFI16 is essential for its cytoplasmic translocation, inflammasome and IFN-beta responses. PLoS Pathog. 11, e1005019 (2015).

26. Dunphy, G. et al. Non-canonical activation of the DNA sensing adaptor STING by ATM and IFI16 mediates NF-KB signaling after nuclear DNA damage. Mol. Cell 71, 745-760.e5 (2018).

27. Thompson, M. R. et al. Interferon $\gamma$-inducible protein (IFI) 16 transcriptionally regulates type I interferons and other interferon-stimulated genes and controls the interferon response to both DNA and RNA viruses. J. Biol. Chem. 289, 23568-23581 (2014)
28. Cao, L. et al. P200 family protein IFI204 negatively regulates type I interferon responses by targeting IRF7 in nucleus. PLoS Pathog. 15, e1008079 (2019).

29. Chang, X. et al. IFI16 inhibits porcine reproductive and respiratory syndrome virus 2 replication in a MAVS-dependent manner in MARC-145 cells. Viruses https://doi.org/10.3390/v11121160 (2019).

30. Kim, B. et al. Discovery of widespread host protein interactions with the pre-replicated genome of CHIKV using VIR-CLASP. Mol. Cell 78, 624-640 (2020).

31. Monzon-Casanova, E. et al. The RNA-binding protein PTBP1 is necessary for B cell selection in germinal centers. Nat. Immunol. 19, 267-278 (2018).

32. Grellscheid, S. N. et al. Molecular design of a splicing switch responsive to the RNA binding protein Tra2 $\beta$. Nucleic Acids Res. 39, 8092-8104 (2011).

33. Li, H. et al. RNA helicase DDX5 inhibits reprogramming to pluripotency by miRNA-based repression of RYBP and its PRC1-dependent and -independent functions. Cell Stem Cell 20, 462-477.e6 (2017).

34. Herdy, B. et al. Analysis of NRAS RNA G-quadruplex binding proteins reveals DDX3X as a novel interactor of cellular G-quadruplex containing transcripts. Nucleic Acids Res. 46, 11592-11604 (2018).

35. Di Giammartino, D. C. et al. RBBP6 isoforms regulate the human polyadenylation machinery and modulate expression of mRNAs with AU-rich 3' UTRs. Genes Dev. 28, 2248-2260 (2014).

36. Li, T., Diner, B. A., Chen, J. \& Cristea, I. M. Acetylation modulates cellular distribution and DNA sensing ability of interferon-inducible protein IFI16. Proc. Natl Acad. Sci. USA 109, 10558-10563 (2012).

37. Baum, A., Sachidanandam, R. \& Garcia-Sastre, A. Preference of RIG-I for short viral RNA molecules in infected cells revealed by next-generation sequencing. Proc. Natl Acad. Sci. USA 107, 16303-16308 (2010).

38. Jakobsen, M. R. \& Paludan, S. R. IFI16: at the interphase between innate DNA sensing and genome regulation. Cytokine Growth Factor Rev. 25, 649-655 (2014).

39. Li, D. et al. STING-mediated IFI16 degradation negatively controls type I interferon production. Cell Rep. 29, 1249-1260 (2019).

40. Merkl, P. E. \& Knipe, D. M. Role for a filamentous nuclear assembly of IFI16, DNA, and host factors in restriction of herpesviral infection. mBio https:// doi.org/10.1128/mBio.02621-18 (2019).

41. Antiochos, B., Matyszewski, M., Sohn, J., Casciola-Rosen, L. \& Rosen, A. IFI16 filament formation in salivary epithelial cells shapes the anti-IFI16 immune response in Sjogren's syndrome. JCI Insight https://doi.org/10.1172/ jci.insight.120179 (2018).

42. Morrone, S. R. et al. Cooperative assembly of IFI16 filaments on dsDNA provides insights into host defense strategy. Proc. Natl Acad. Sci. USA 111, E62-E71 (2014).

43. Hotter, D. et al. IFI16 targets the transcription factor Sp1 to suppress HIV-1 transcription and latency reactivation. Cell Host Microbe 25, 858-872 (2019)

44. Thapa, R. J. et al. DAI senses influenza a virus genomic RNA and activates RIPK3-dependent cell death. Cell Host Microbe 20, 674-681 (2016).

45. Kuriakose, T. et al. ZBP1/DAI is an innate sensor of influenza virus triggering the NLRP3 inflammasome and programmed cell death pathways. Sci Immunol. https://doi.org/10.1126/sciimmunol.aag2045 (2016).

46. Sui, H., Zhou, M., Chen, Q., Lane, H. C. \& Imamichi, T. siRNA enhances DNA-mediated interferon lambda-1 response through crosstalk between RIG-I and IFI16 signalling pathway. Nucleic Acids Res. 42, 583-598 (2014).

47. Cao, L. et al. The nuclear matrix protein SAFA surveils viral RNA and facilitates immunity by activating antiviral enhancers and super-enhancers. Cell Host Microbe 26, 369-384 (2019).

48. Gao, Q. et al. A cell-based high-throughput approach to identify inhibitors of influenza A virus. Acta Pharm. Sin. B 4, 301-306 (2014).

49. Wei, F. et al. Induction of PGRN by influenza virus inhibits the antiviral immune responses through downregulation of type I interferons signaling. PLoS Pathog. 15, e1008062 (2019).

50. Manicassamy, B. et al. Analysis of in vivo dynamics of influenza virus infection in mice using a GFP reporter virus. Proc. Natl Acad. Sci. USA 107, 11531-11536 (2010).

51. Gavazzi, C. et al. An in vitro network of intermolecular interactions between viral RNA segments of an avian $\mathrm{H} 5 \mathrm{~N} 2$ influenza A virus: comparison with a human H3N2 virus. Nucleic Acids Res. 41, 1241-1254 (2013).

52. You, F. et al. ELF4 is critical for induction of type I interferon and the host antiviral response. Nat. Immunol. 14, 1237-1246 (2013).

53. Zhang, Y. et al. Identifying local and descending inputs for primary sensory neurons. J. Clin. Invest. 125, 3782-3794 (2015).

54. Perez-Riverol, Y. et al. The PRIDE database and related tools and resources in 2019: improving support for quantification data. Nucleic Acids Res. 47, D442-D450 (2019).

\section{Acknowledgements}

We thank F. You (Peking University), W. Liu (Institute of Microbiology, Chinese Academy of Sciences), Y. Zhu (Wuhan University) and Y. Chen (Wuhan University) for kindly providing cell lines, and W. Tang (Shandong University) for generously gifting the 
p204 $4^{-/-}$mice. This work was supported by the National Key Research and Development Program of China (2016YFD0500204 to J.L.) and the National Natural Science Foundation of China (81960297 to F.W.).

\section{Author contributions}

Z.J. and F.W. performed and analysed most of the experiments. Y.Z., T.W. and W.G. performed the AP-MS experiments. S.Y., H.S., J.P., Y.S., M.W. and Q.T. generated biochemical reagents. C.G. and K.-C.C. guided and analysed the data. F.W. and J.L. conceived and supervised the study.

\section{Competing interests}

The authors declare no competing interests.

\section{Additional information}

Extended data is available for this paper at https://doi.org/10.1038/s41564-021-00907-x. Supplementary information The online version contains supplementary material available at https://doi.org/10.1038/s41564-021-00907-x.

Correspondence and requests for materials should be addressed to F.W. or J.L.

Peer review information Nature Microbiology thanks Peter Staeheli, Aartjan te Velthuis and the other, anonymous, reviewer(s) for their contribution to the peer review of this work. Peer reviewer reports are available.

Reprints and permissions information is available at www.nature.com/reprints.

Publisher's note Springer Nature remains neutral with regard to jurisdictional claims in published maps and institutional affiliations.

(c) The Author(s), under exclusive licence to Springer Nature Limited 2021 

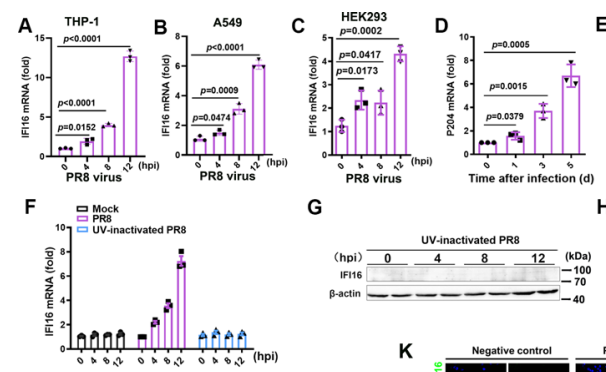

G

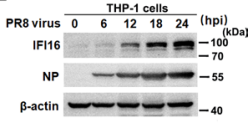

G

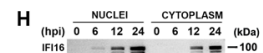
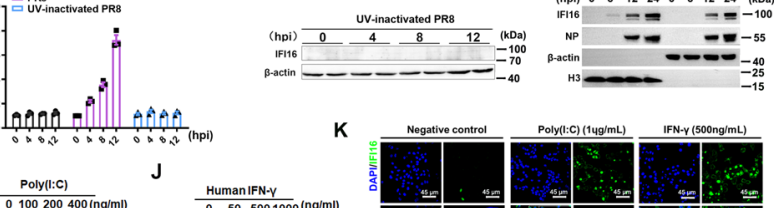

$\mathrm{J}$

$\frac{\text { Poly(l:C) }}{0100200400 \text { (ng/mll) }}$

$\mathrm{J}$

Human IFN- $\gamma$

K

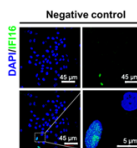

Polyl:: (1) (19g/m!

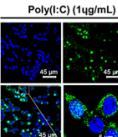

IFN.r (500ng/mL)
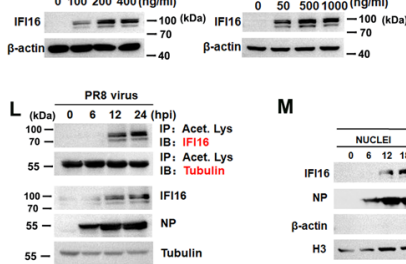

M

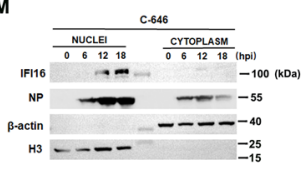

Extended Data Fig. 1 | IFI16 induction by IAV is dependent on viral replication. a-c, IFI16 expression in a, THP-1, b, A549, and c, HEK293 was quantified by RT-qPCR. (d) p204 expression in lung tissues from PR8 virus-infected WT mice was determine. e, IFl16 expression in $1.0 \mathrm{MOI}$ of PR8 virus-infected THP-1 cells was detected. f, IFI16 mRNA expression in $2 \mathrm{MOI}$ of UV-inactivated and live PR8 virus-infected A549 cells was determined. $\mathbf{g}$, IFI16 protein expression in $2 \mathrm{MOI}$ of UV-inactivated PR8 virus-infected A549 cells was determined. $\mathbf{h}$, IFl16 expression in the nuclear and cytoplasmic fractions of PR8 virus-infected A549 cells were determined. $\beta$-actin and H3 were used as purity markers for cytoplasmic and nuclear fractions, respectively. $\mathbf{i}$, IFI16 expression in A549 cells transfected with poly (I:C) for $18 \mathrm{~h}$ was determined by Western blotting. $\mathbf{j}$, IFI16 expression in A549 cells treated with IFN- $\gamma$ for $18 \mathrm{~h}$ was determined. (k) Intracellular localization of IFI16 in A549 cells treated with poly(I:C) or IFN- $\gamma$ for $12 \mathrm{~h}$ was determined. Scale bars, $45 \mu \mathrm{m}$ and $5 \mu \mathrm{m}$ (enlarged). I, A549 cells were infected with PR8 virus at 0, 6, 12 and 24 hpi. Cell lysates were then immunoprecipitated with anti-acetylated lysine. Bound proteins were analyzed by immunoblots with anti-IFI16 antibody. m, A549 cells were pre-incubated with C-646 for $2 \mathrm{~h}$, then infected with PR8 virus for $1 \mathrm{~h}$, washed and incubated in complete medium with or without C-646. IFI16 expression and viral NP protein in the nuclear and cytoplasmic fractions of PR8 virus-infected $\mathrm{A} 549$ cells at indicated time points were determined by Western blotting. $\beta$-Actin and $\mathrm{H} 3$ were used as purity markers for cytoplasmic and nuclear fractions, respectively. (a-d) and $\mathbf{f}$, Data presented as means \pm SD from three independent experiments. (e) and $\mathbf{g}-\mathbf{m}$, Data are representative of three independent experiments. Statistical significance in (a) to (d) and (f) was determined by unpaired two-tailed Student's t-test. 


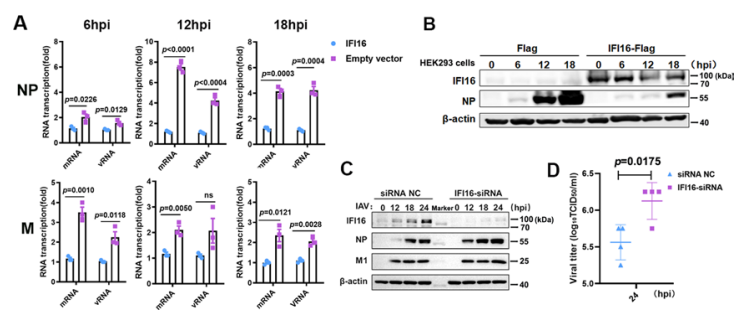

Extended Data Fig. 2 | IFI16 inhibits IAV viral replication. a, A549 cells were transfected with IFl16-Flag plasmids or empty control for $24 \mathrm{~h}$ and then infected with PR8 virus at $2.0 \mathrm{MOI}$. mRNA and vRNA expression of NP and M genes at 6, 12 and 18 hpi were determined by RT-qPCR. Data are presented as means \pm SEMs from three independent experiments. b. HEK293 cells were transfected with IFI16-Flag plasmids or empty control for $24 \mathrm{~h}$ and then infected with PR8 virus at $2.0 \mathrm{MOI}$. NP protein expression at $0,6,12$ and $18 \mathrm{hpi}$ was determined by Western blotting. $\beta$-Actin detection was used as loading control. (c) and (d), A549 cells were transfected with IFl16-targeting siRNA and negative control (NC) siRNA for $24 \mathrm{~h}$, followed by infection with PR8 virus $(\mathrm{MOI}=1)$. c, NP and M1 protein expression were determined by Western blotting at $0,12,18$ and $24 \mathrm{hpi}$. $\beta$-Actin detection was used as loading control. d, Viral titers were determined by TCID50 assay at the $24 \mathrm{hpi}$. Data presented as means \pm SD from three independent experiments. (b) and (c), Data are representative of three independent experiments. Statistical significance in (a) and (d) was determined by unpaired two-tailed Student's t-test. ns $=$ non-significant. 


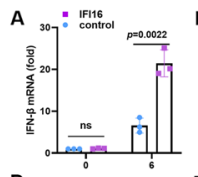

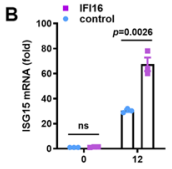

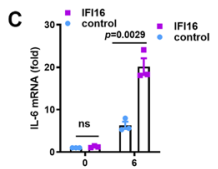

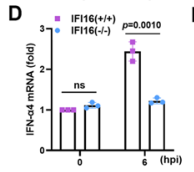

E

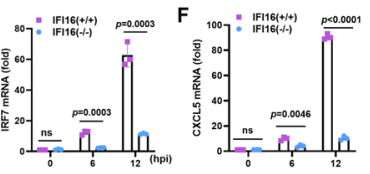

G

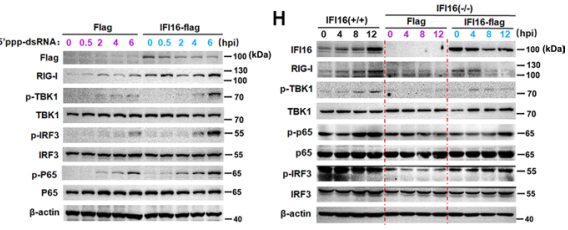

Extended Data Fig. 3 | IFI16 enhances the activation of IFN-I pathway during IAV infection. a to c, Serum-starved A549cells were transfected with IFI16-encoding plasmids or negative control, and then infected with $1.0 \mathrm{MOI}$ of PR8 virus. Expression of IFN- $\beta$ (a), ISG15 (b), IL-6 (c) at the indicated time points was determined by RT-qPCR. $\mathbf{d}$ to $\mathbf{f}$, mRNA expression of IFN- $\alpha 4$ (d), IRF7 (e) and CXCL5 (f) in PR8 virus-infected IFI16+/+ and IFI16-/- A549 cells at 0, 6, and 12 hpi was determined by RT-qPCR. (g) Immunoblot analysis of RIG-I-triggered downstream signaling pathway in IFI16+/+ and IFI16-/A549 cells after stimulation with 5'ppp-RNA for the indicated duration. h, IFI16+/+ and IFI16-/- A549 cells transfected with IFI16-Flag expression vectors or empty control for $24 \mathrm{~h}$ and then infected with PR8 virus at $1.0 \mathrm{MOI}$. RIG-I-triggered downstream signaling pathway at 0, 4, 8 and $12 \mathrm{hpi}$ was assessed with indicated antibodies. a to $\mathbf{f}$, Data presented as means \pm SD from three independent experiments. $\mathbf{g}$ to $\mathbf{h}$, Data are representative of three independent experiments. Statistical significance in (a) to (f) was determined by unpaired two-tailed Student's t-test. ns $=$ non-significant. 


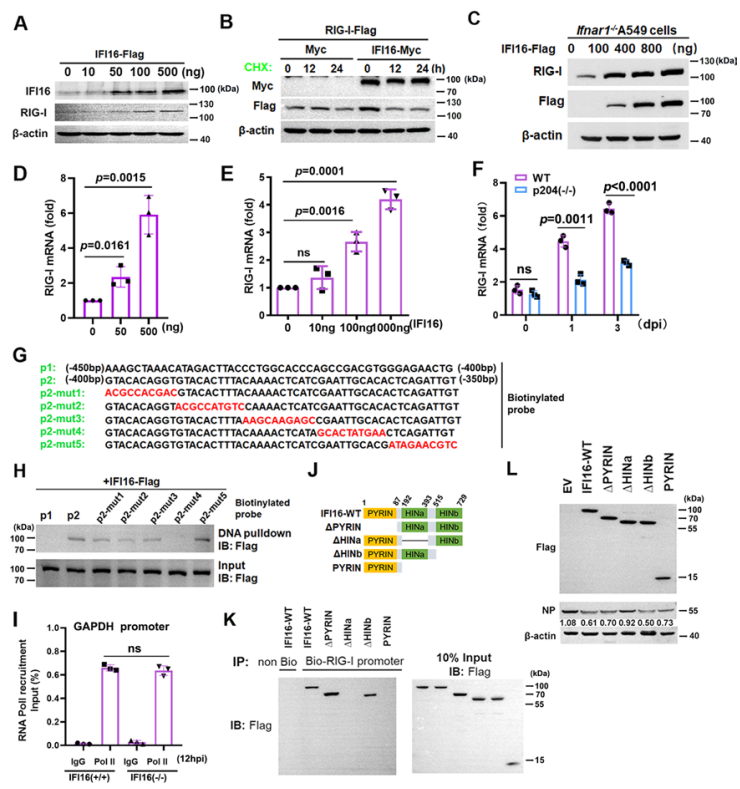

Extended Data Fig. 4 | IFI16 binds RIG-I promoter and enhances RIG-I transcription. a, RIG-I expression in A549 cells transfected with IFI16-Flag vectors was examined. b, HEK293 cells co-transfected with indicated plasmids for $24 \mathrm{~h}$ were treated with cycloheximide (CHX) for 12 and $24 \mathrm{~h}$. Expression of RIG-I and IFI16 in cell lysates was detected. c, RIG-I expression in ifnar1-/-A549 cells transfected with IFI16-Flag vectors was examined. d, RIG-I mRNA expression in ifnar1-/- A549 cells transfected with IFI16-Flag plasmids for $24 \mathrm{~h}$ was determined. e, RIG-I expression in A549 cells transfected with IFI16-Flag plasmids for $24 \mathrm{~h}$ was determined. f, RIG-I expression in lung tissues from virus-infected WT and p204-/ - mice $(n=3)$ was determined. $\mathbf{g}$, Schematic diagram of the biotinylated probe sequences from the promoter of RIG-I gene and corresponding mutants. $\mathbf{h}$, IB analysis of the binding ability between wild type (p1 and p2) and mutated (p2-mut1 to p2-mut5) probes, and IFI16 proteins. i, IFI16+/+ and IFI16-/-A549 cells were infected with PR8 virus for $12 \mathrm{~h}$, followed by ChIP assay. $\mathbf{j}$, Schematic diagram of full-length IFI16 vectors and truncated mutants. $\mathbf{k}$, A549 cells were transfected with Flag-tagged full-length IFI16 vectors and truncated mutant plasmids. After $24 \mathrm{~h}$ transfection, nuclear extracts were incubated with non-biotinylated or biotinylated promoter sequence of RIG-I for $4 \mathrm{~h}$. Nuclear extracts were examined for IFI16 and truncated mutant expression by Western blotting. I, A549 cells after Flag-tagged IFI16 vectors and truncated mutants transfection and PR8 infection was determined. $\mathbf{d}$ to $\mathbf{f}$ and $\mathbf{i}$, Data are representative of three independent experiments (mean \pm SD). (a), (b), (c), (h), (k), and (I), Data are representative of three independent experiments. Statistical significance in (d) to (f) and (i) was determined by unpaired two-tailed Student's t-test. ns = non-significant. 

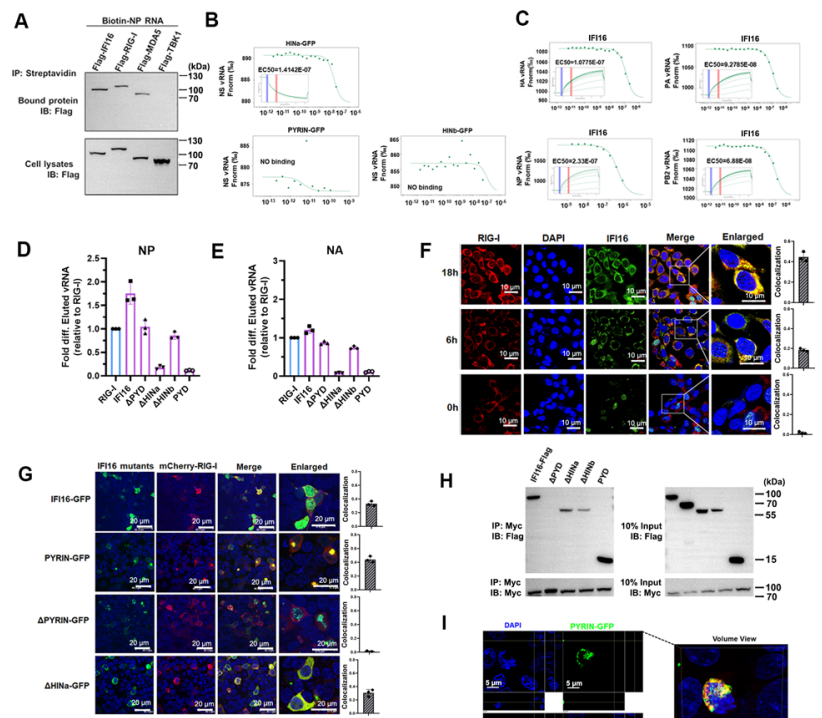

H

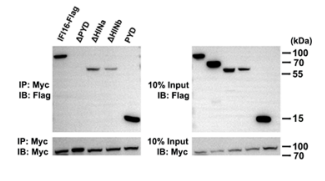

I

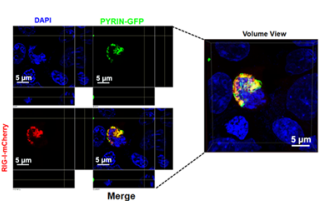

Extended Data Fig. 5 | IFI16 directly binds viral RNA during infection. a, HEK293 cells were transfected with indicated vectors for 24 h. Cell lysates were incubated with biotin-labeled viral NP RNA and immunoprecipitated with streptavidin beads. Bound proteins were analyzed by immunoblots with anti-Flag antibody. b, HEK293 cells were transfected with HINa-GFP, HINb-GFP, and PYRIN-GFP expression vectors for $24 \mathrm{~h}$. Cell lysates were incubated with influenza NS vRNA. Binding affinity between indicated proteins and NS vRNA was determined by MST assays. c, Purified GST-IFI16 proteins were incubated with fluorescein labeled influenza HA, NP, PA and PB2 vRNAs. Binding affinity was determined by MST assays. $\mathbf{d}$, PCR detection of NP vRNA in eluted RNA from RIG-I-Flag, IFI16-Flag and indicated Flag-tagged IFI16 truncated constructs. The data represent means \pm SD. ( $n=3$ independent experiments). e, PCR detection of NA vRNAs in eluted RNA from RIG-I-Flag, IFI16-Flag and indicated Flag-tagged IFl16 truncations. Data were normalized to vRNA from RIG-I-Flag immunoprecipitates. The data represent means \pm SD. ( $n=3$ independent experiments). $\mathbf{f}$, Confocal microscopy detecting the co-localization of endogenous IFI16 and RIG-I in PR8 virus-infected A549 cells at 0,6 and 18 hpi. Nuclei were stained with DAPI (left). Scale bars, $10 \mu \mathrm{m}$. Quantification of the co-localization of IFI16 and RIG-I in cells (right). Means \pm SD from 3 biological samples. g, Confocal microscopy of HEK293 cells transfected with RIG-I-mCherry plasmids, together with expression vectors for IFI16-GFP or GFP-tagged mutants. DAPI serves as a marker for nuclei (left). Scale bars, $20 \mu \mathrm{m}$. Quantification of the co-localization of IFI16 or mutants and RIG-I in cells (right). Means \pm SD from 3 biological samples. h, Co-IP analysis of the interaction between Myc-tagged RIG-I and Flag-tagged full-length or mutant IFI16 in HEK293 cells. (i) Three-dimensional confocal microscopy of HEK293 cells co-transfected with plasmids encoding PYRIN-GFP and RIG-I-mCherry. DAPI serves as a marker for nuclei. All data are representative of three independent experiments. Scale bars, $5 \mu \mathrm{m}$. 


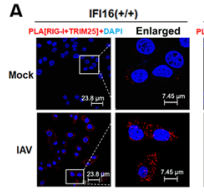

c

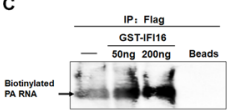

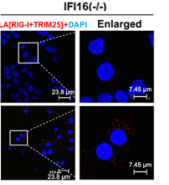

D

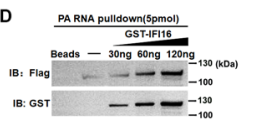

IB: GST

F
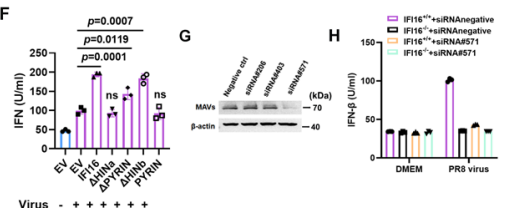

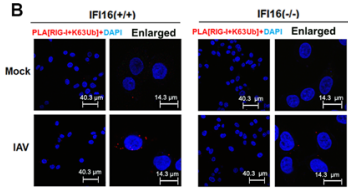

E

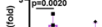

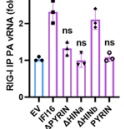

$\sin 2000$

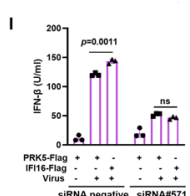

Extended Data Fig. 6 | RIG-I is involved in IFI16-mediated antiviral response in IAV infection. a, IFI16+/+ or IFI16-/- A549 cells were infected with PR8 virus $(\mathrm{MOI}=1)$ and then analyzed by PLA. The right panels are enlarged. Red point represents TRIM25 plus RIG-I complexes. Scale bars, $23.8 \mu \mathrm{m}$ (left) and $7.45 \mu \mathrm{m}$ (enlarged). b, IFI16+/+ or IFI16-/- A549 cells were infected with PR8 virus (MOI=1) and then analyzed by PLA. The right panels are enlarged. Red point indicates K63 Ub plus RIG-I complexes. Scale bars, $40.3 \mu \mathrm{m}$ (left) and $14.3 \mu \mathrm{m}$ (enlarged). c, RIP-EMSA analysis of the binding of biotinylated PA vRNA to purified Flag-RIG-I protein by adding purified GST-IFI16 at dose of 50 and 200 ng. d, RNA pull-down analysis of the binding of biotinylated PA vRNA to purified Flag-RIG-I protein by adding purified GST-IFI16 at dose of 30, 60, and 120 ng. e, A549 cells were transfected with Flag-tagged IFI16, its truncated forms and control. RNA eluted from RIG-I immunoprecipitates were detected by RT-qPCR with specific primers of PA gene. f, A549 cells were transfected with Flag-tagged IFI16 and its truncated forms for $24 \mathrm{~h}$ and then infected with PR 8 virus. Production of IFN- $\beta$ in supernatants at 18 hpi was determined. $\mathbf{g}$, Knockdown effect of MAVS-targeting siRNAs in A549 cells. h, IFI16+/+ or IFI16-/- A549 cells were transfected with control or MAVS-targeting siRNA \#571 and after $24 \mathrm{~h}$ infected with PR8 virus. Production of IFN- $\beta$ in supernatants at 18 hpi was determined (i) IFl16+/+ A549 cells were transfected with negative control or MAVS-targeting siRNA \#571, and $24 \mathrm{~h}$ later, cells were transfected with Flag-IFl16 plasmids or control; $24 \mathrm{~h}$ later cells were infected with PR8 virus. Production of IFN- $\beta$ in supernatants on 18 hpi were quantified. (e), (f), (h), and (i), Data are representative of three independent experiments (mean \pm SD). (a) to (d) and ( $\mathbf{g}$ ), All data are representative of three independent experiments. Statistical significance in (e) to (f) and (i) was determined by unpaired two-tailed Student's t-test. ns = non-significant. 
A

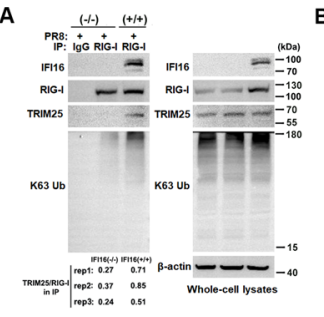

B

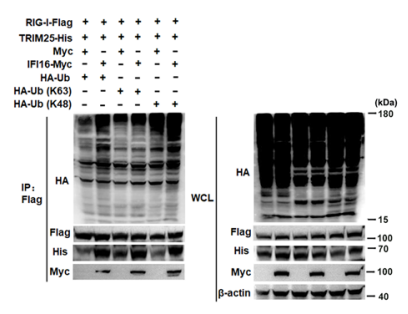

C

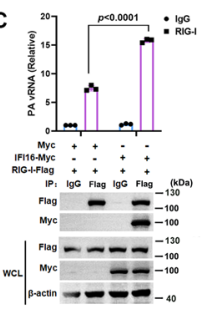

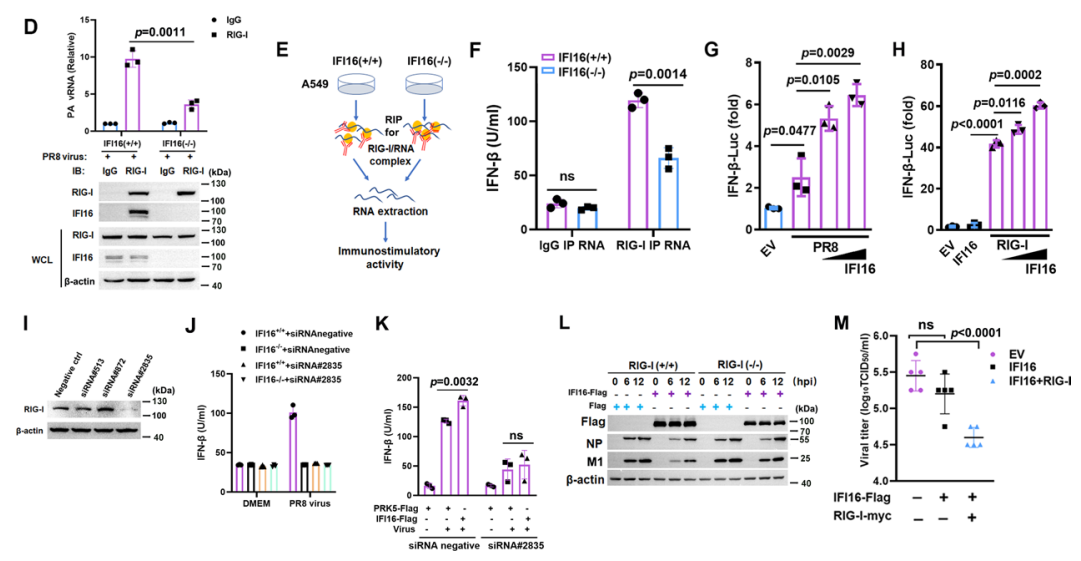

Extended Data Fig. 7 | IFI16 promotes RIG-I signaling. a, The interaction between TRIM25, RIG-I, and K63-linked ubiquitination of RIG-I in PR8 virus-infected IFI16+/+ and IFI16-/- A549 cells. b, Co-IP analysis of RIG-I ubiquitination in HEK293 cells transfected with indicated plasmids. c, Cell lysates were prepared after indicated plasmids transfection and PR8 infection and then immunoprecipitated with control lgG or anti-Flag. Bound-RNA was extracted for qPCR analysis. d, IFI16+/+ and IFI16-/- A549 cells were infected with PR8 virus for $12 \mathrm{~h}$. Cell lysates were then immunoprecipitated with IgG or anti-RIG-I. Bound-RNA was extracted for analysis. e, Schematic experimental procedure used in (f). f, RNA co-purified with RIG-I from IAV-infected IFI16+/+ and IFI16-/- A549 cells were transfected into A549 cells, and IFN- $\beta$ was determined. $\mathbf{g}$, Luciferase activity of HEK293 cells after transfection with IFI16 vectors or control and then infection with PR8 virus. h, Luciferase activity of HEK293 cells transfected with an IFN- $\beta$ reporter plasmids, RIG-I vectors, and control or IFI16 vectors. i, Knockdown effect of RIG-I-targeting siRNAs in A549 cells. j, IFN- $\beta$ in IFI16+/+or IFI16-/- A549 cells after transfection with siRNA \#2835 or control and infection with PR8 virus. k, IFN- $\beta$ in IFI16+/+ cells after transfection with siRNA \#2835 or control, IFI16-Flag or control plasmids, and infection with PR8 virus. I, NP protein levels in RIG-I-/- HEK293 cells after transfection with IFI16-Flag vectors or control and infection with PR8 virus were determined. $\mathbf{m}, \mathrm{RIG}-\mathrm{I}-$ /- HEK293 cells were transfected with indicated plasmids for $24 \mathrm{~h}$ and then infected with PR8 virus at $1.0 \mathrm{MOI}$. Viral titers were determined. (a) to (d), (i), and (I), Data are representative of three independent experiments. (c) to $(\mathbf{d}),(\mathbf{f})$ to $(\mathbf{h}),(\mathbf{j}),(\mathbf{k})$ and $(\mathbf{m})$, Data presented as means \pm SD from three independent experiments, and the significance of the results was assessed using a parametric paired t-test (Student's two-tailed t-test). ns = non-significant. 


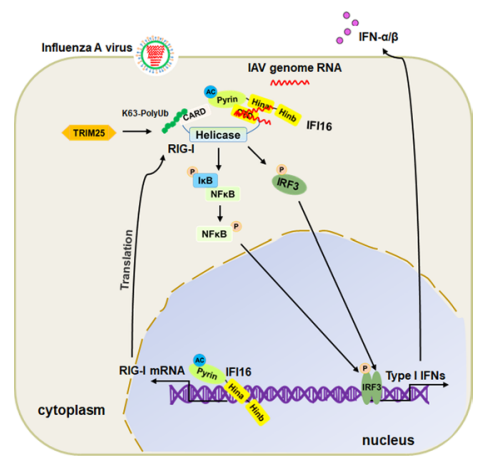

Extended Data Fig. 8 | Schematic model to show IFI16 enhances RIG-I signaling in influenza virus infection. Briefly, influenza virus infection upregulates IFI16 expression; IFI16 protein, in turn, enhances transcription of RIG-I. In addition, IFI16 protein, via its HINa domain, directly senses viral RNA and, via its PYRIN domain, interacts with RIG-I receptor to promote antiviral RIG-I signaling. 


\section{Reporting Summary}

Nature Research wishes to improve the reproducibility of the work that we publish. This form provides structure for consistency and transparency in reporting. For further information on Nature Research policies, see our Editorial Policies and the Editorial Policy Checklist.

\section{Statistics}

For all statistical analyses, confirm that the following items are present in the figure legend, table legend, main text, or Methods section.

n/a Confirmed

$\square \bigotimes$ The exact sample size $(n)$ for each experimental group/condition, given as a discrete number and unit of measurement

$\square$ \A statement on whether measurements were taken from distinct samples or whether the same sample was measured repeatedly

$\square$ The statistical test(s) used AND whether they are one- or two-sided

$\square$ Only common tests should be described solely by name; describe more complex techniques in the Methods section.

Х $\square$ A description of all covariates tested

$\square$ \A description of any assumptions or corrections, such as tests of normality and adjustment for multiple comparisons

$\square$ A full description of the statistical parameters including central tendency (e.g. means) or other basic estimates (e.g. regression coefficient)

$\bigotimes$ AND variation (e.g. standard deviation) or associated estimates of uncertainty (e.g. confidence intervals)

$\square$ For null hypothesis testing, the test statistic (e.g. $F, t, r$ ) with confidence intervals, effect sizes, degrees of freedom and $P$ value noted

Give $P$ values as exact values whenever suitable.

Х $\square$ For Bayesian analysis, information on the choice of priors and Markov chain Monte Carlo settings

Х $\square$ For hierarchical and complex designs, identification of the appropriate level for tests and full reporting of outcomes

\ $\square$ Estimates of effect sizes (e.g. Cohen's $d$, Pearson's $r$ ), indicating how they were calculated

Our web collection on statistics for biologists contains articles on many of the points above.

\section{Software and code}

Policy information about availability of computer code

Data collection The Imaris 9.2 platform Microsoft 2010

GraphPad Prism version 7.00

NanoTemper analysis tool

Data analysis The Imaris 9.2 platform

Microsoft 2010

GraphPad Prism version 7.00

NanoTemper analysis tool

For manuscripts utilizing custom algorithms or software that are central to the research but not yet described in published literature, software must be made available to editors and reviewers. We strongly encourage code deposition in a community repository (e.g. GitHub). See the Nature Research guidelines for submitting code \& software for further information.

\section{Data}

Policy information about availability of data

All manuscripts must include a data availability statement. This statement should provide the following information, where applicable:

- Accession codes, unique identifiers, or web links for publicly available datasets

- A list of figures that have associated raw data

- A description of any restrictions on data availability 


\section{Field-specific reporting}

Please select the one below that is the best fit for your research. If you are not sure, read the appropriate sections before making your selection.

$\searrow$ Life sciences

$\square$ Behavioural \& social sciences

Ecological, evolutionary \& environmental sciences

For a reference copy of the document with all sections, see nature.com/documents/nr-reporting-summary-flat.pdf

\section{Life sciences study design}

All studies must disclose on these points even when the disclosure is negative.

Sample size No sample-size calculation was performed. Luciferase reporter assays and qPCR experiments have been performed at least 3 times. Western blot assays were performed at least 3 times. Virus titers were examined for each sample, $n=3$, with at least a triplicate for each experiment.

Data exclusions No data were excluded from the analyses.

Replication All attempts at replication were successful.

Randomization All samples were randomly assigned to experimental groups. All mice experiments were randomized

Blinding The investigators were blinded to group allocation for animal experiments, including virus inoculation, tissue collection, virus titer analysis, and evaluation of histological changes.

\section{Reporting for specific materials, systems and methods}

We require information from authors about some types of materials, experimental systems and methods used in many studies. Here, indicate whether each material, system or method listed is relevant to your study. If you are not sure if a list item applies to your research, read the appropriate section before selecting a response.

Materials \& experimental systems

\begin{tabular}{l|l}
\multicolumn{2}{l}{ Methods } \\
\hline n/a & Involved in the study \\
$\bigotimes$ & $\square$ ChIP-seq \\
$\varnothing$ & $\square$ Flow cytometry \\
$\bigotimes$ & $\square$ MRI-based neuroimaging
\end{tabular}

\begin{tabular}{|c|c|}
\hline $\mathrm{n} / \mathrm{a}$ & Involved in the study \\
\hline & $\bigotimes$ Antibodies \\
\hline & $\bigotimes$ Eukaryotic cell lines \\
\hline$\bigotimes$ & Palaeontology and archaeology \\
\hline & $\bigotimes$ Animals and other organisms \\
\hline Х & Human research participants \\
\hline Х & Clinical data \\
\hline Х & Dual use research of concern \\
\hline
\end{tabular}

\section{Antibodies}

Antibodies used

Anti-IFI16 (ab185812), anti-TBK1 (ab40676), anti-NP (ab104870), anti-TBK1 (ab40676), anti-Ubiquitin (linkage-specific K63) (ab271929), anti-NP (ab20343), anti-acetyl Lysine (ab190479) and anti-RNA polymerase II (ab5095) antibodies were from Abcam. Anti-M1 (GTX125928) antibody were from GeneTex. Anti- $\beta$-actin (\#AA128) antibody were from Beyotime Biotechnology. Anti-IFI16 (sc-8023) and anti-TRIM25 (sc-166926) antibodies were from Santa Cruz. Rabbit anti-p65 (\#10745-1-AP), anti-HA (\#66006-2-Ig) and anti-RIG-I (\#20566-1-AP) antibodies were from Proteintech. Anti-IRF3 (YT2398) antibody was from ImmunoWay Biotechnology Company. Anti-Phospho-TBK1 (Ser172; \#5483); anti-Phospho-IRF3 (Ser396; \#4947), anti-IFI16 (\#14970S), anti-FLAG (\#8146), anti-His (\#9991), anti-GST (\#2624), anti-Histone H3 (\#4499), anti-Tubulin (\#3873), anti-MAVs (24930), anti-Myc (\#2278); anti-Phospho-p65 (Ser536; \#3033) antibodies were from Cell Signaling Technology Inc.;

Validation

All the commercial antibodies have been verified by the manufactures according to the western blot assays and images from their websites.

\section{Eukaryotic cell lines}

\section{Policy information about cell lines}

Cell line source(s)

Madin-Darby canine kidney cells (MDCK), human embryonic kidney cells (HEK293), and human lung adenocarcinoma epithelial cells (A549) were maintained in-house. THP-1 cell line was kindly provided by Dr. Shijun Zheng (China Agricultural University). IFI16 KO (IFI16-/- A549) and RIG-I KO (RIG-I-/- HEK293) cell lines were generously given by Dr. Yu Chen (Sun Yatsen University) and Dr. Wenjun Liu (Institute of Microbiology, Chinese Academy of Sciences), respectively. Ifnar1 KO (ifnar1-/- 


\section{Authentication}

Mycoplasma contamination

Commonly misidentified lines (See ICLAC register)
A549) cell line was generously given by Dr. Ying Zhu (Wuhan University). And 2fTGH-ISRE cell line (human fibrosarcoma cells expressing an ISRE driven luciferase reporter) was kindly provided by Dr. Fuping You (Peking University)31. HEK293T-Gluc cells were kindly provided by Dr. Shan Cen (Chinese Academy of Medical Sciences and Peking Union Medical School).

MDCK, HEK293, A549, and THP-1 cells were verified by ATCC. Cell morphology was monitored at each passage by microscope. We will discard the cells after 15-20 passages, and recover new cells from frozen stocks.

All cell lines tested negative for mycoplasma contamination.

No commonly misidentified cell lines were used.

\section{Animals and other organisms}

Policy information about studies involving animals; ARRIVE guidelines recommended for reporting animal research
Laboratory animals
6- to 8-week-old male and female C57BL/6 mice.

Wild animals

The study did not involve wild animals

Field-collected samples

The study did not involve samples collected from the filed

Ethics oversight

All animal experiments were performed in accordance with institutional guidelines of China Agricultural University (CAU) (approval SKLAB-B-2010-003) and approved by the Beijing Association for Science and Technology of China (approval SYXK, Beijing, 2007-0023).

Note that full information on the approval of the study protocol must also be provided in the manuscript. 